\title{
Color and Variability Characteristics of Point Sources in the Faint Sky Variability Survey
}

\author{
Mark E. Huber ${ }^{1}$ \\ Lawrence Livermore National Laboratory \\ P.O. Box 808, L-413, Livermore, CA, USA \\ huber9@llnl.gov \\ Mark E. Everett ${ }^{1}$ \\ Planetary Science Institute \\ 620 N. 6th Ave., Tucson, AZ, USA \\ and \\ Steve B. Howell ${ }^{1}$ \\ WIYN Observatory \& NOAO \\ 950 N. Cherry Ave., Tucson, AZ 85726, USA
}

\begin{abstract}
We present an analysis of the color and variability characteristics for point sources in the Faint Sky Variability Survey (FSVS). The FSVS cataloged $\sim 23$ square degrees in $B V I$ filters from $\sim 16-24$ mag to investigate variability in faint sources at moderate to high Galactic latitudes. Point source completeness is found to be $>83 \%$ for a selected representative sample $(V=17.5$ $22.0 \mathrm{mag}, B-V=0.0-1.5)$ containing both photometric $B, V$ detections and $80 \%$ of the timesampled $V$ data available compared to a basic internal source completeness of $99 \%$. Multiepoch (10-30) observations in $V$ spanning minutes to years modeled by light curve simulations reveal amplitude sensitivities to $\sim 0.015-0.075 \mathrm{mag}$ over a representative $V=18-22 \mathrm{mag}$ range. Periodicity determinations appear viable to time-scales of an order 1 day or less using the most sampled fields ( $\sim 30$ epochs). The fraction of point sources is found to be generally variable at $5-$ $8 \%$ over $V=17.5-22.0 \mathrm{mag}$. For $V$ brighter than $19 \mathrm{mag}$, the variable population is dominated by low amplitude $(<0.05 \mathrm{mag})$ and blue $(B-V<0.35)$ sources, possibly representing a population of $\gamma$ Doradus stars. Overall, the dominant population of variable sources are bluer than $B-V=0.65$ and have Main Sequence colors, likely reflecting larger populations of RR Lyrae, SX Phe, $\gamma$ Doradus, and W UMa variables.
\end{abstract}

Subject headings: surveys (FSVS) - stars: statistics, variables - Galaxy: stellar content

\footnotetext{
${ }^{1}$ Visiting astronomers at the Isaac Newton Telescope
} 


\section{Introduction}

Static, wide-field population studies continue to progress to fainter limits (e.g., SDSS to $g^{\prime}=21$ mag; Chen et al. 2001) and continue to produce a better understanding to the Galactic boundaries and beyond. Recently, wide-field, temporal surveys have begun to catch up, going to deeper limits and becoming increasingly more common, such as the BSVS (Everett et al. 2002), QUEST (Vivas et al. 2004), SuperMACHO, (Becker et al. 2004), and the FSVS (Groot et al. 2003), with a variety of different science goals and target populations, probing new areas in astrophysics. A survey like the FSVS with variability sampling over hours to years samples a large range of dynamical phenomena including mass transfer events, pulsations, and stellar activity with amplitudes $\sim 0.01$ and larger. Thus, the FSVS with multi-band photometry and variability sampling provides for both multiple population studies and for independent comparisons to other surveys.

For the FSVS to meet its design goals and be useful to future programs, an understanding of the distribution of colors and variability sampled by the FSVS is necessary. A representative region of magnitude and color space was selected to provide a typical completeness level that can be achieved in the overall dataset. Simulations of the variability sampled by the FSVS were produced in order to probe the sensitivity of the FSVS data. Using the FSVS dataset, a sub-sample was selected and examined for the general distribution of sources across magnitude, color, and variability space as a preliminary study.

Section 2 briefly reviews the key properties of the FSVS dataset described in detail by Groot et al. (2003) and describes the field sample selections used as the basis in the following sections with completeness estimates. The resulting color space is described is Section 3, including the extreme color limits and comparison to star counts of a Bahcall Galactic model. The variability space is described in section 4 including detection sensitivity in time-scale and amplitudes. Section 5 combines the color and variability information to investigate the variable nature of point source populations, both galactic and extragalactic, in terms of a variability fraction by color. Section 6 presents a discussion and summary of the dataset and study.

\section{FSVS Observations and Dataset}

The FSVS provides a database for studying faint populations and their variability, spanning $\sim 16-25$ mag across the optical regime using the $B, V$, and $I$ filters ${ }^{1}$ with time-sampling obtained in the $V$-band over 10 's of minutes to years. With a total areal coverage of $\sim 23$ square degrees at moderate to high Galactic latitudes, the FSVS also provides a sample spanning relatively different Galactic populations. The FSVS was designed as both a photometric and astrometric survey to investigate the general potential of such a survey to faint magnitudes, Kuiper Belt Objects, intrinsically faint ultracool dwarfs and old white dwarfs, interacting binaries, RR Lyrae stars, and optical transients to gamma-ray bursts. Since its completion, additional studies including white dwarf-red dwarf binaries, quasars (lensing, variability, and association with galaxy groups) and galaxy clusters (Soc̈hting et al. 2006) have been added. In comparison to other surveys, the FSVS may only have a fraction of the areal coverage and time-sampling of the all-sky variability surveys, but the FSVS proceeds several magnitudes fainter opening up a distinct and significant volume of space.

The data outputs of the survey include processed $B, V$, and $I$ images and four photometric databases

\footnotetext{
${ }^{1}$ Standard Harris $B, V$ and RGO $I$ filter set at the Isaac Newton Telescope, http://www.ing.iac.es/ quality/filter/filt4.html. The wavelength coverages (center/width) for each filter are $4298 / 1065 \AA, 5425 / 975 \AA$, and 8063/937 ̊ for $B, V$, and $I$ respectively.
} 
with astrometry. The four photometry methods include variable PSF fitting, 2xFWHM aperture, fixed 12 pixel aperture, and isophotal magnitudes, where the variable PSF fitting photometry is the recommended dataset for variability studies. The stellarity parameter from SExtractor provides point-to-extended source discrimination in the $B$ and $V$ filters, but due to the thinned CCDs $I$ is highly inconsistent. In using a stellarity measure determined from co-adding the bulk of the best $V$ images, point-to-extended source discrimination reaches to $V=23.5-24$ mag overall. However, to depths fainter than $V=22-23$, many point sources are mis-classified as extended objects. The absolute calibration of magnitudes is $\sim 0.05$ and $\sim 0.1$ for $B V$ and $I$ respectively and astrometric positions are found to be reliable to $\sim 0.5^{\prime \prime}$ over the entire dataset. The FSVS public release data ${ }^{2}$ as described by Groot et al. (2003) is used in this work. An additional version of the dataset will be available in the near future at the NOAO Data Products Program web site ${ }^{3}$ and updates there will be described in future works as they are utilized.

\subsection{Field Groups and Sample Completeness}

Like all general surveys, the FSVS data set is not inherently uniform or complete (e.g., field pointing offsets, poor weather, technical problems). The unavoidable non-uniform sampling will not interfere with the general design of the FSVS to sample a large range of variability as described in Groot et al. (2003). However, an estimation of the degree of incompleteness is required in order to cope with possible inherent selection effects and biases. To investigate the incompleteness, sub-sets in the FSVS data were created incorporating similar observational properties of magnitude, color, and variability sensitivities. In this paper we present a summary of the completeness using two specifically constructed field groups that are representative of the entire set as a whole.

The FSVS field groups presented in Table 1 are spatially contiguous fields that will share similar observational properties - from the same observing run, similar Galactic populations, and similar time-sampling properties. Field group 01 and 19 are similar in time-sampling and areal coverage, but are from different observing seasons and sample potentially different stellar populations at mid- to high latitudes respectively. Field Group 25 is similar in Galactic position to Field Group 19 and observed in the same observing run, but has a larger sample of observations over an observing week, fewer yearly re-observations, and smaller areal coverage.

For our purposes here, we have initially selected the dynamic range in the FSVS to be set by the saturation limit at the bright end and a $3 \sigma$ detection with respect to the background sky flux at the faint end of the calibrated dataset. The completeness of this sample should not depend on the parameter to obtain a set of point sources (i.e., the stellarity). As shown in Figure 7 of Groot et al. (2003), the stellarity value for the classification of point sources (stellarity $>0.8$ ) is usable to $V$ of $23-24$ mag, but begins to degrade beyond $V \sim 22$ mag. Therefore, a $V$ range will need to be chosen to restrict losses due to point-to-extended source confusion.

Figure 1 presents histograms of all sources contrasted with point sources detected in the $B, V$, and $I$ filters that have no error flag in field groups 01 . The source number peaks at 23.8, 23.4, and 21.5 mag in $B, V$, and $I$ respectively. The $B$ and $V$ filters show little change other than a loss of extended and fainter sources, moving the number peak to 23.6 and 22.4 mag in $B$ and $V$ respectively. The $I$ filter shows

\footnotetext{
${ }^{2}$ http://staff.science.uva.nl/ fsvs/programs/README

${ }^{3}$ http://www.noao.edu/dpp
} 
a significant deviation in the number of sources beyond $19.5 \mathrm{mag}$. The additional loss of sources in the $I$ filter is due to the required association for classification of a point source on the detection of the source in the $V$ filter (see Section 3.1 on extreme colors). With the $I$ filter detection result, further investigation of completeness will thus be done using only the $B$ and $V$ detections. In order to provide a uniform, complete sample over all field groups and provide a significant range of astrophysical importance, a representative $V$ magnitude range was adopted. Table 2 summarizes the magnitude limits and the slight differences between the FSVS field groups samples.

At fainter magnitudes, the profile of fainter stars becomes significantly noisy and may become contaminated by crowding with faint galaxies in the wings of the star causing a mis-classification of the true point source as an extended one. Conversely, de-blended galaxy sets may be classified more as point-like, adding to the confusion. To determine the magnitude threshold for clear classification of point sources, a number fraction is investigated as a function of magnitude shown in Figure 2. Two number fraction cases are plotted, the number of sources with a stellarity value $>0.9$ divided by the total number of sources $>0.8$ and the number of sources with stellarity $>0.8$ divided by the number of sources with stellarity $>0.6$. Both cases show a similar fraction indicating a similar trend of point sources moving to uncertain extended values of stellarity at fainter magnitudes. At $V \sim 22 \mathrm{mag}$, the point near which the slope of the trend becomes much steeper, $\sim 93 \%$ of the sources are still considered strongly point-like. By $V \sim 23$ mag, the stellarity measure has lost many sources to the uncertain extended classification as there are roughly equal numbers being distributed throughout the stellarity range.

Losses from contamination factors including truncation at and near CCD edges, cosmic rays and bad pixels, close and bright neighbors, and saturation artifacts must also be quantified. As the adjoining fields observed overlap by a few arcminutes, source duplication is possible. Thus, sources with matching coordinates to $<1^{\prime \prime}$ are checked for and the data with less time-sampling and poorer magnitudes are removed. An additional requirement has been applied to the sample that at least $80 \%$ of the total $V$ observations exist, chosen to allow for the occasional loss by small field shifts into a bad pixel or inner CCD edge, and cosmic ray influence, but to not degrade the variability sampling. Losses due to close, bright neighbors and saturation artifacts are estimated by visual inspection of the images for point sources $V<22.0$ mag. The loss of sources that fall in between CCDs is not an issue as the total areal size was calculated using the sum for each CCD. The adopted $V$ bright limit of 17.5 avoids losses due to variation in the saturation level. Table 3 summarizes the average loss of sources due to the factors mentioned above. Inclusion of a possible $7 \%$ loss due to mis-classified point sources contributes approximately one-third to nearly half in the completeness estimate losses. The requirement that $80 \%$ of the time-sampled $V$ measurements exist is found to be of negligible effect in the completeness estimate. The dominant degradation of completeness comes from the simultaneous requirement of a $B$ and $V$ measurement, with $7-10 \%$ of sources lost due to detection losses in $B$ (see Sect. 3.1).

With the multi-epoch observations, an estimation for the probability of a missed detection can be used as an internal check of completeness for a single detection as used in the Palomar-Green Survey (Green et al. 1986). The fraction of a single detection to a double detection is given

$$
\frac{N_{1}}{N_{2}}=\frac{a}{b}=\frac{2 p(1-p)}{p^{2}}=\frac{2}{p}-2
$$

where $N_{1}$ and $N_{2}$ are the number of sources found once and twice respectively, making $p$ the probability of only a single detection. The completeness can then be estimated using the percent of duplicate sky coverage by

$$
\text { completeness }=(1-d) p+d\left[2 p(1-p)+p^{2}\right]
$$


where $d$ is the fraction of duplicate coverage. In the FSVS, an average of $96 \%$ with variations $<1 \%$ was found for the overlapping coverage and used for $d$. All fields groups were found to have a high level of internal completeness using any of the 12-30 epochs in combination. Within the adopted magnitude range, the possible sources of detection loss are combined and show a nearly uniform estimate of total completeness presented in Table 3 for each field group.

\section{Color Space}

Exploration of the FSVS color space provides a view of the populations contained within the FSVS. Figure 3 presents an example description of the $B-V, V-I$ color-color space and source number histograms in $V$ magnitude groups. The color limits given in Table 2 still define a complete sample for $V=17.5-22$ mag. The $V-I$ color is included, but is not considered complete to the levels in Table 3 except for the first two $V$ magnitude group sets, 17.5-19.0 and 19.0-20.5. For Main Sequence dwarfs, the $B-V$ color range spans the early A (A0) to early M (M2-M5) spectral types, and also reaches late K to M0 giants as well (Cox 2000). However, with just broadband $B V I$ filters and color calibration uncertainties of $0.07(B-V)$ and $0.11(V-I)$ in addition to the photometric uncertainties, color separation between luminosity classes is not possible. Also, no correction is made for extinction or reddening in the sources as the correction is small, by design of the field selection (Groot et al. 2003) process. This along with other details will be investigated in an overall encompassing analysis in a later work. The set of extreme color sources, sources not detected in all 3 filters, is presented in the following sub-section. The $V$ magnitude groups illustrate the changing population contributions to the color space sample in the FSVS field groups with a a bimodal distribution in the $B-V$ color space. The contribution to the redder $B-V$ colors increases dramatically as additional cooler dwarfs were sampled at fainter magnitudes. In the $V=22-23$ mag group, the distribution is strongly smeared by the increasing color uncertainty as shown in the $B-V, V-I$ color-color plot.

\subsection{Extreme Color Space}

Ideally, extreme color sources are sources that do not have a complete detection in all filters due to having a steep spectral energy distribution. As the observations in multiple filters are not simultaneous, it is possible for sources with variability or proper motions to show up as extreme color sources. The extreme color sources may also be junk, or more commonly, image detection limited sources. False extreme color sources will provide an idea of the quality of source detection, matching, or inherent problems in the dataset pipeline itself. Thus, image detection limited sources provides yet another test of the quality and limiting magnitude output from the pipeline reduction process.

With three filters used in the FSVS, there are six possible extreme color source types; $B, V$, and $I$ only detections and combinations of $B V, V I$ and $B I$. In terms of their spectral energy distribution, possible types of sources that will show up in $B$ only may include very hot sources such as hot white dwarfs and accretion powered objects. At longer wavelengths, $I$ only detections can include cooler temperature sources such as very low-mass dwarfs (investigated in more detail along with with $V I$ detections by cross-matching the brightest with 2MASS photometry and follow-up spectroscopy (Huber et al. 2006a)). $V$ only detections could include odd spectral sources such as quasars with strong emission (i.e., $L \alpha$ ) redshifted into the $V$ band region (causing at least a $B$ band dropout) or even further into the $I$ band resulting in an $I$ only detection. Detection in the two filters $B V$ and $V I$ can be sources with less extreme of a spectral energy distribution 
(i.e., white dwarfs and $\mathrm{M}$ dwarfs), and more so near the detection limits. Detection of a source in $B I$ will be just as extreme as a detection of $V$ only just in the opposite sense with emission shifted out of the $V$ bandpass or more into the $B I$ bandpasses such as composite objects like possibly white dwarf-red dwarf binaries. However, many extreme color sources are likely to be just near the image detection limits. Table 4 provides the color limits for sources in order to produce an extreme color boundary and give an average detection limit of the CCDs in the field group 01 (e.g., for a $B$ only detection at the image limit, the $B-V$ color must be less than or equal to 0.52). The work of Zaggia et al. (1999) presents a useful comparison of populations in the BVI color space that when combined with the FSVS extreme color boundaries reveals where sources can appear as extreme color sources in the FSVS.

Variable or transient sources can also be recorded as single filter detections or filter dropouts given that the observations in the different filters are not simultaneous. Inclusion of the multiple $V$ observations into a mean $V$ to replace the sources with missing photometric $V$ during the $V B I V$ color sequence is possible, but not done here as sources can be largely variable and non-periodic requiring a case by case investigation. Timescales of order 15 mins separate the different filter observations in the $V B I V$ color sequence and up to 1 year in the $V$ observation that defines an exclusion window for detection. This timescale spans a wide range of populations that will exist near the detection limit of the survey (i.e., $M$ dwarfs, quasars, supernovae, orphan GRB afterglows). Some may show up once in one filter (i.e., fast outburst flare on an active M dwarf) during the color sequence or in $V$ at the peak of their variability and possibly $B$ or $I$ depending on the spectral energy distribution (i.e., $B$ band dropouts in the case of $M$ dwarfs again).

Single filter detections may also include rapidly moving solar system sources. A preliminary investigation by a team at the Planetary Science Institute (Neese 2002) looking for KBOs resulted in no conclusive identifications. Since the field groups are far from the ecliptic, the numbers here are expected to be small. Two moving sources were clearly identified in the $V$ only detection group that were bright enough to be classified as point-like, and are likely to be from the larger population of Main Belt asteroids.

The error flags from the FSVS pipeline do not report information on the null detection of a source making it necessary to investigate the extreme color sources on a source by source basis. The total numbers of extreme color sources for field group 01 is given in Table 4 as a typical set. The occurrence of junk sources, ones that should have been detected and were not, is also estimated as a percentage of the total number cataloged. The sources of junk are identified as sources that were mis-matched, sources lost near saturated sources from bleeding and spikes, and sources affected by cosmic rays and prior unlabeled bad pixels ${ }^{4}$. For sources with a $V \leq 23.0$ detection, the stellarity measure was utilized to give a percentage of the sources that are classified as point-like in Table 4.

No ideal extreme color sources were detected at the bright magnitude end of the survey, only near or at the faint detection limit. Overall, the mid- and high galactic latitude fields are similar in their content of extreme color sources and thus FSVS field group 01 is presented as a representative sample from the numbers presented in Table 4. Histograms of single filter detections are shown in Figure 4 and show the majority of the $B$ and $V$ only detections occur at the plate limits. However, the $I$ only detection sources show an enhanced number well above the plate limit of the survey and partially explain the decline of sources detected as point sources in Figure 1 with very red limiting colors that peaks at $\geq 2.6$ (populations of cooler, low-mass dwarfs and high redshift quasars). Histograms of two filter detections are shown in Figure 5 . The histograms illustrate that the majority of the extreme color sources are actually just sources at the plate

\footnotetext{
${ }^{4}$ In addition, some junk sources were also still detected from the vignetted corner (NE corner of CCD 3; Groot et al. 2003), but are not included in the numbers in Table 4.
} 
limits of the survey. Figure 6 shows histograms of the point sources with $V \leq 23$ mag for the $B V$ and $V I$ detections. The population missing as shown in Figure 1 are identified as the $V I$ detected sources with $V>23$ mag.

\subsection{Galactic Stellar Model Comparison}

The simple two component Galaxy model (main disk and halo) of Bahcall \& Soneira (1986) was used to compare the populations in field groups 01 and 19. As the FSVS sources only have BVI photometry with fairly large color uncertainties (of order $0.1 \mathrm{mag}$ ), separation of luminosity classes is impossible. The Bahcall model was run using the parameters listed in Table 5 to produce a simple description of the expected distributions of stars. M13 was used as the halo turnoff as it provides generally the best fit to high galactic latitude observations (Bahcall \& Soneira 1984). Even though stars earlier than spectral type A are not expected, the bright absolute magnitude $\left(\mathrm{M}_{v}\right)$ was set at -6 as the function is smooth and will reflect the negligible low population. The faint $\mathrm{M}_{v}$ was set at 16.5 to provide a comparison to prior population studies (i.e., Bahcall \& Soneira 1986), but may be an uncertain result since the FSVS is only complete to $B-V \sim 1.5$ and for dwarfs to $M_{v} \sim 12.5$. The color binning in the model output is similar to the uncertainties of the FSVS photometry. Table 6 gives the output numbers of stars and percentage of giants for the entire sample, the disk, and the halo. Figure 7 presents the FSVS star counts as a histogram of $B-V$ color with the Bahcall model result over-plotted. The lower latitude field group 01 shows the larger number of disk stars at redder $B-V$ in comparison to the spheroid contribution. The high latitude field group 19 shows a roughly equal bimodal distribution with the spheroid contribution being larger for the bluer $B-V$ peak and the disk contribution being larger for the redder $B-V$ peak. However, the models do not exactly match the FSVS populations. Red-ward of 1.5 in $B-V$, both FSVS field groups show an under-abundance that is a reflection of the incompleteness of the samples for redder sources. Blue-ward of $B-V=0.5$, both the FSVS field groups are also under-abundant and may indicate an incompleteness problem as well. In FSVS field group 01, an over-abundance from $B-V \sim 0.6-1.4$ is likely a thick disk contribution (Chen et al. 2001) not included in the Bahcall model. FSVS field group 19 shows no strong overabundance until $B-V \sim 1.2-1.4$ due to a much lower number contribution as expected from the disk population. Using the outputs from the Bahcall model, an estimate of the number of giants in the field groups can be made $(\sim 8 \%$ and $13 \%$ at mid- and high galactic latitudes respectively) that will be useful for understanding the population of variable sources detected in the FSVS until better photometry is obtained (e.g., source cross-matching to the SDSS). Table 6 breaks down the numbers of expected giants for the field groups.

\section{Variability Detection and Sensitivity}

With the FSVS time-sampling, a general statistical test must be applied to test for variability. For the detection of variability, there are three thresholds that must be considered; the probability in a statistical test, the amplitude, and the time-sampling. The first threshold is a pure statistical result, while the latter two will be related to the type of variability present. However, all three parameters will be convoluted by the others and simulations were constructed to test and explore the potential of the FSVS data set for detection of variability. The determination of variability will also be affected by the level of variability with respect to the photometric uncertainties and the number of points sampled and are included in the simulations. The large time-sample set can be broken up into smaller time groups to investigate intranight, day, week, and month-year variability. To compare the variability, the FSVS field groups have been made roughly similar 
as summarized in Table 7. Field groups 01 and 19 have the same number of nights (3), and yearly reobservations (2), with slightly different number of observations within each night, particularly in field group 01. Field group 25 has observations over 6 nights with only a 1 year re-observation. Field group 01 and 19 represent the earlier time-sampling method in the FSVS, while Field group 25 represents the switch to a larger sampling set within a week observing run. The two time-sampling groups, 01 and 25 , were specifically investigated to understand the benefits gained by additional time-sampling. Field groups 01 and 19 were found to be equivalent.

To generally determine if a source is variable, an evaluation of the probability that the deviations in a light curve are consistent with the photometric errors was made to provide a null-hypothesis test. Violation of the null-hypothesis test indicates variability in the source. To determine if the deviation in a light curve is consistent with the photometric errors, a reduced $\chi^{2}\left(\chi_{\nu}^{2}\right)$ value is calculated with respect to a weighted mean magnitude. The weighted mean magnitude is given by

$$
<m_{w}>=\frac{\sum^{N}\left(m_{i} / \sigma_{i}^{2}\right)}{\sum_{i}^{N}\left(1 / \sigma_{i}^{2}\right)}
$$

where $N$ is the number of included observations in the mean, $m_{i}$ is the $i^{\text {th }}$ magnitude, $\sigma_{i}$ is the $i^{\text {th }}$ photometric error. The probability in the $\chi_{\nu}^{2}, \mathrm{P}\left(\chi_{\nu}^{2}\right)$, is the calculated integral of the probability distribution function for $\chi^{2}$. Essentially, the $\mathrm{P}\left(\chi_{\nu}^{2}\right)$ is a measure of the probability that another set of measurements on the same source would yield a light curve of equal or greater $\chi^{2}$ in the fit. Thus, if $\mathrm{P}\left(\chi_{\nu}^{2}\right)$ of the light curve is 0.01 , a confidence level of $99 \%$ can be ascribed, taken to be that the source is very likely variable. Statistically, a confidence level of $99 \%$ also indicates that only 1 in 100 sources showing similar variations would be found variable due to random fluctuations and not true variability in the source. If the uncertainties were not appropriately accounted for or if largely deviant photometry points exist (i.e., cosmic ray hit), spurious detections of variability would result as was found in the $2 \mathrm{xFWHM}$ aperture photometry in the first case (see Figure 6; Groot et al. (2003)). Violation of the null test to a threshold $\mathrm{P}\left(\chi_{\nu}^{2}\right)$, or confidence level, would indicate that the light curve shows some form of variability.

The $\mathrm{P}\left(\chi_{\nu}^{2}\right)$ has no dependence on time directly, only the number of sample points and amplitude variations with respect to the photometric uncertainties. To investigate the variability at different time-scales, the sample was divided into time groups; night, week, week with nightly averages, all data with the week averaged. The $\mathrm{P}\left(\chi_{\nu}^{2}\right)$ was then calculated separately for the separate time groups. There is also the occurrence that nightly or yearly time groups may have only 2 photometric points. In the case of only two sample points, the number of sigma separation of the points can be used, but is not simulated here. Table 8 summarizes the re-sampling time groups of the FSVS photometry. The time groups offer only a guideline to the variability time-scale sampled as will be shown in the following section on simulations. The true amplitude of the variability is also difficult to clearly assess with the sparse and incomplete time-sampling. Thus, the amplitude of variability was determined simply by the $\Delta \mathrm{V}$ peak-to-peak values for the time group.

\subsection{Variability Simulations}

A set of FORTRAN routines was developed for simulation of light curves to model FSVS data and determine an appropriate threshold for classifying a source as variable. The program allows for inclusion of the field sampling pattern and photometric uncertainties to a basic sinusoidal function. The FSVS field groups used were found to have similar photometric uncertainties for the run of time-sampled data, mostly equivalent to a $\sqrt{\left.<\sigma^{2}\right\rangle}$ value for the uncertainties or the measured uncertainties at each epoch. Additionally 
added to the simulated time-sampled photometry was a Gaussian distribution of noise to represent an unmeasured variation present in the data set. A standard deviation amplitude based on the half-width at half-maximum of the observed $\sigma_{l c}$ distribution in each field group was used for each $V$ magnitude group, $\sim 0.33 \sigma_{V}$.

A set of 10000 non-variable light curves was created for the $V$ magnitudes 18, 20, and 22 for field group 01 and 25. Table 9 presents a summary of the statistical test results in a percent of the fraction falsely detected as variable at $\mathrm{P}\left(\chi_{\nu}^{2}\right)$ thresholds ranging from $10^{-1}$ to $10^{-4}$ and the re-sampled time groups for an average. The magnitude range dependence over $V=18-22$ was weak and thus incorporated into the average uncertainties. The false variability detections are obviously higher than statistically expected due to the added Gaussian noise for the under-represented photometric uncertainty in the photometry. With the inclusion of the additional noise term, the false detection percentage from the non-variable light curve simulations give a truer measure of the false detections expected in the FSVS light curves. As a significant effect is not found in comparing FSVS field group 01 and 25 with difference sample sizes, a similar threshold of $\mathrm{P}\left(\chi_{\nu}^{2}\right)$ is useful for any FSVS field.

A set of 10000 variable light curves for each field group was generated using a sinusoidal function to the non-variable configuration described above to test the sensitivity of the FSVS to detect variability. The periods ranged from 12 minutes to 600 days, similar to possible periods detectable by the FSVS and having scientific significance (e.g., flickering in accretion processes and g-mode pulsations in white dwarfs, to long period variables). The test amplitudes ranged from 1-10 times the photometric uncertainty in order to cover the possible sensitivity limits imposed by the photometry over the magnitude range. Figure 11 presents a sample of simulated light curves from field group 01 and 25 for key periods to demonstrate the time-sampling provided and what null level and $10 \sigma$ variability appears to the eye. Figure 8 illustrates the detected fractions with a $\mathrm{P}\left(\chi_{\nu}^{2}\right) \leq 10^{-2}$ for field group 01 and Figures 9 and 10 are the detected fractions with a more stringent $\mathrm{P}\left(\chi_{\nu}^{2}\right) \leq 10^{-4}$. for field groups 01 and 25 . The 2 points for the All, Week Ave sample in field group 25 are not shown, but were found similar, with the larger time-scale, to a $\sigma$ separation measure for two points within a night. The light curve simulations indicate that nightly and week with night average samples provide a good possibility of clearly detecting shorter time-scale variability. From all the observations having a minimum 12 hour daily duty cycle, detection of day time-scale variability is degraded. Longer time-scales ( $>1$ day) are not possible to clearly isolate from shorter time-scale variability here with these test groups. No significant difference is evident in the slightly different sampling of the field groups 01 and 19. Overall, the larger number of photometric epochs also provides a slightly better chance of detecting variability to lower amplitudes.

The simulated light curves were also investigated for potential detection of periodicity using the Phase Dispersion Minimization (PDM) routine (Stellingwerf 1978). PDM is not strongly dependent on using sinusoids to detect periodicity and can accommodate unevenly-sampled data well. The PDM program was run to evaluate periods in FSVS field group 01 and 25, a minimum and maximum number epoch sample set in the FSVS. The results of the period search are shown in Figure 12 for a sample of short periods where a lower theta represents a more significant period and a $95 \%$ confidence level is illustrated. The lower sampled FSVS field group (01) thetagram is mostly dominated by noise and may only present a very general idea of possible periods. Longer ( $>1$ day) and shorter $<90 \mathrm{~min}$ ) periods show even less distinct levels of detectable periodicity, essentially limiting potential period determinations to the order of 1 hour to 1 day using the higher sampled FSVS fields. 


\section{Variability Fractions by Magnitude and Color}

To study the general relation of variability to magnitude and color, a variability detection threshold must be selected. From previous studies of low level variability (Grenon 1993; Everett et al. 2002), a $<10 \%$ fraction is expected to be found as a function of $V$ magnitude. As there are $\sim 2000$ to upwards of 10000 sources typical in the FSVS field groups, 200-1000 sources are expected to have variability detected. Thus, to limit the number of false detections to $\sim 1 \%$ and less for $200-1000$ sources, a $\mathrm{P}\left(\chi_{\nu}^{2}\right) \leq 10^{-4}$ is required. The percentage of variable sources not detected will then depend on the variability amplitude and timescale in the light curve. The difference in the sensitivity to variability from $\mathrm{P}\left(\chi_{\nu}^{2}\right) \leq 10^{-2}$ to $\mathrm{P}\left(\chi_{\nu}^{2}\right) \leq 10^{-4}$ is roughly a factor of 2 in relation to the amplitude threshold. The difference in false detections, however, is greatly reduced by a factor of 10 or more in going from a $\mathrm{P}\left(\chi_{\nu}^{2}\right) \leq 10^{-2}$ to $\mathrm{P}\left(\chi_{\nu}^{2}\right) \leq 10^{-4}$ threshold. A tighter $\mathrm{P}\left(\chi_{\nu}^{2}\right)$ threshold also constrains the sensitivity of the short time-scale variability tests. Using a $\mathrm{P}\left(\chi_{\nu}^{2}\right) \leq 10^{-4}$ threshold for detection, the FSVS field groups from the prior sections were tested for variability on the different test sampling and in $V$ magnitude groups. Figures 13-16 present the final results in the form of a variability fraction of the number of sources detected with variability, $N_{v}$, divided by the total number of sources, $N$, in a $V$ magnitude or $B-V$ color bin. The $V$ magnitude bin range is $V=17.5-22.0$ (covering the completeness percentage given in Table 3 ) and including the less complete (though point source classifiable range) $V=22.0-23.0$ in $1.5 \mathrm{mag}$ bin steps. The color bin range covers $B-V$ of -0.1 to 2.3 in bin steps of 0.3 , but only using $V=17.5-22.0 \mathrm{mag}$. The uncertainty in variability fraction $\left(\sigma_{N_{v} / N}\right)$ of each magnitude and color bin is given by formal propagation of errors assuming the uncertainties in $N$ and $N_{v}$ follow simple Poisson counting statistics. The $\sigma_{N_{v} / N}$ becomes

$$
\sigma_{N_{v} / N}=\frac{\sqrt{N_{v}\left(1+N_{v} / N\right)}}{N}
$$

where $N$ is the total number of sources and $N_{v}$ is the number of sources that are identified as variable in a magnitude or color bin.

The sample was investigated with respect to the different time sample groups. The groups with 2 points were not included due to their lower confidence and likely require inspection by eye to confirm they are not problematic. The sample was also divided into amplitude groups to investigate the variability fraction as a function of the $\Delta V$ peak-to-peak changes with $\Delta V<0.05 \mathrm{mag},<0.1 \mathrm{mag},<0.5 \mathrm{mag}$, and $<1.0$ mag. Figures 13 and 14 present the variability fraction as a function of $V$ magnitude for the two FSVS field groups 01 and 19. FSVS field group 25 was found to be much noisier due to having a smaller sample of point sources and was not investigated further (see Huber 2002). The variability over the entire FSVS sample $V$ range shows a maximum variability fraction of 7-8\% overall in both mid- and high galactic latitude fields. The decline in variability fraction toward the faint end of the magnitude range may simply be due to the increasing photometric uncertainty and thus decreased sensitivity to all but the largest amplitude variables. In the mid-galactic field group 01, the majority of the brighter $V$ sources were found to vary at the $<0.05$ magnitude level with a possible indication of a more rapid decline due to loss of sensitivity of the photometry. However, the photometric uncertainties are still $\sim 0.01 \mathrm{mag}$ to $V=20 \mathrm{mag}$ and the light curve simulations indicate variability is detectable to amplitudes $\sim 0.03 \mathrm{mag}$ unless the time-scale is on the order of a day or 10s of days. A similar, though weaker, feature is seen in FSVS field group 19 (high latitude) where the amplitude level $<0.1 \mathrm{mag}$ is more significant. Variability on time-scales of a week only is found in field groups 01 and 19 to the 3-6\% level, with the higher latitude field 19 showing a higher percentage of variability. Variability detected within a night is low at $\sim 1 \%$ and only at larger amplitudes, $<0.5$ mag. In the mid-latitude field group, the fraction is nearly zero until after $V \sim 19$ mag. However, the high latitude field 19 shows a mostly uniform, though noisier, sample at 1\%. In comparison to Everett et al. (2002), who 
found an overall variability fraction of $1 \%$ in their week-long sample, the fraction here is 3-4 times in field groups 01 and 19. However, the two fraction are roughly equivalent if the amplitude level for variability is limited to the photometric uncertainties in the Everett et al. (2002) study.

Figures 15 and 16 show the variability fraction of sources for the field groups as a function of $B-V$ color for the most complete magnitude sample $V=17.5-22.0$. A significant variability fraction is found for $B-V<0.65$ in the month-yearly time-scale, up-wards of $40 \%$ in both latitude fields with amplitudes $<0.5$ mag. In all field groups, the week only time-scale variability fraction is found to be $\sim 1 \%$ for a $B-V=0.65-1.5$ with amplitudes $<0.5 \mathrm{mag}$, but field group 19 shows a peak to $8 \%$ for bluer colors. Variability on time-scales within a night is found in $\sim 1-2 \%$ of the sources over the entire $B-V$ range, except in field group 01 where the variability fraction peaks at $B-V \sim 0.35$ to $5 \%$. Field group 19 also appears to have a peak in the variability fraction at $B-V \sim 0.05$, but with large uncertainties. Going even fainter, no significant result is seen in the $V=22.0-23.0$ mag range, similar to $V=20.5-22.0$ mag range, but with fewer sources and is not shown.

With the addition of $I$ magnitudes, when available, the majority of variable sources follow the stellar locus for $B-V, V-I$ color space. With an estimate of 580 and 465 giants from the Bahcall model (8 and $14 \%$ for field group 01 and 19 respectively, or $27 \%$ and $22 \%$ for the spheroid specifically), the majority of the blue region could likely be populated by those in the Instability Strip and composed of pulsating giants. The peak in the variability fraction for the bluer sources is also similar to that found by Everett et al. (2002) with variability within a night, identified to be populated with $\delta$ Scuti/SX Phe stars.

\section{Discussion and Summary}

The FSVS color and variability space has been investigated to determine its usefulness and potential. In using the survey for point-source population studies, the stellarity or point-to-extended discriminator begins to degrade in completeness beyond $V=22 \mathrm{mag}$, but can and will be used to 23-24 mag for future studies on specific populations of variables. The completeness of the FSVS depends strongly on the chosen parameters: number of filters for a detection, the color range, and the number of $V$ follow-up observations required. The internal completeness of the survey is estimated to be $>99 \%$. However, for a range in $V=17.5-22.0 \mathrm{mag}$, range in $B-V$ of $0.0-1.5$, and at least $80 \%$ of the $V$ epochs present, the completeness level drops to a possible minimum of $83-85 \%$ for the fields investigated. Extreme color sources tend to either be junk detections or sources at the imaging plate limit except for $V I$ and $I$ detections where a large number are represented by the cooler population of dwarfs at the end of the Main Sequence and high redshift quasars (Huber et al. 2006a). The results using a Bahcall \& Soneira (1986) Galaxy model show the expected distribution of main disk and halo population of stars. The Galaxy model fits at bluer and redder $B-V$ colors indicate a possible loss of completeness at the color boundaries. In the mid-latitude field group, a thick disk contribution appears to be present for $B-V 0.6$ to 1.4 and in the high latitude field an abundance of sources are found in $B-V$ of 1.2-1.4. The Bahcall model predicts $8 \%$ and $13 \%$ of the sources to be giants in the mid- and high latitude fields respectively. In comparison to other color surveys such as the SDSS and EIS, the FSVS does poorly with the small number of filters and larger color uncertainties. However, the SDSS will eventually cover more of the FSVS fields to similar magnitudes in which variability is useful and will thus provide a good color match to the time-sampled sources. The SDSS overlap will be very useful for future studies of specific variable populations (i.e., pulsational variables, AGN/QSOs, cataclysmic variables).

Simulated light curves with no variability and periodic, sinusoidal variability over the magnitude and 
time-scale range of the FSVS were generated to test the levels of false variability detections, the amplitude sensitivity, and time-scale sensitivity. Using a simple null hypothesis test with the probability in a reduced $\chi^{2}$ fit of the light curve to its mean magnitude level, a test for variability in a light curve is achieved. The false detection rate of sources depends on the $\mathrm{P}\left(\chi_{\nu}^{2}\right)$ level and the time group sample of points only, ranging from $2.8 \%$ to $0.1 \%$ for $\mathrm{P}\left(\chi_{\nu}^{2}\right) \leq 10^{-2}$ and $\mathrm{P}\left(\chi_{\nu}^{2}\right) \leq 10^{-4}$ respectively for the entire sample (All group, Table 9 ). An acceptable level of false detections will depend on the sample type and size as discussed in the science topic chapters. No strong dependence on magnitude is found for false detections. The magnitude, due to increasing photometric uncertainty, degrades the amplitude sensitivity levels for detection of variability as shown Figures 8-10. Overall, the amplitude sensitivity ranges from $\sim 0.015-0.075$ mag including all the epoch points over the $V$ magnitude range of the FSVS. The time-scale sensitivity of the FSVS ranges from the shortest time between sequential observations ( $\sim 12$ minutes) to the longest time spacing between observations ( $\sim 1000$ days) as in field group 01. Simulated light curves were generated with periods ranging from 90 minutes to 600 days and show less sensitivity to variability on time-scales on the order of the 24 hour cycle and 30 days and longer as the number of observations become very sparse. The time-sampling in the FSVS fields with $\sim 30$ epochs allows a rough estimation of possibly periodicity for periods of 1-10 hours. The test for periodicity would be enhanced with a larger sample, but at the same time reduce the total areal coverage of the survey. In comparison to other variability surveys such as the BSVS, LONEOS, and MACHO, the FSVS fills a missing niche. The BSVS only goes to $V \sim 19 \mathrm{mag}$, LONEOS only reaches to $R \sim 19 \mathrm{mag}$, and MACHO/SuperMACHO probes specifically different fields and hence different populations. Overlap in the FSVS and LONEOS will be useful to match the brighter FSVS sources and construct a larger set of longer time-sampled observations being careful to take into account the behavior of different variable types in the different survey bandpasses.

The FSVS data set provides a test-bed for time-sampling for future surveys of this kind (e.g., the Largeaperture Synoptic Survey Telescope, LSST; Tyson \& Angel 2001) and methods to optimize the scientific return. A gradient form of time-sampling and a minimum of 25-30 epochs are really necessary as shown by comparing the two FSVS field groups 01 and 25. A large sample, 5-6, observations in one night, followed by a a similar size sample in a neighboring night would greatly benefit the determination of periods on hour time-scales. Adding 2-3 observations throughout the entire observing run would provide a better sample for day time-scales. The primary weakness in the FSVS data set, aside from the 24 hour cycle due to nightly observations from a single site facility, is the weekly time-scale sample as an observing run was only 5-6 days. Adding a following week or month observation set would provide a better sample for week or greater time-scales. Another weakness of this gradient form is the degraded coverage of fast features with long separations (i.e., eclipsing binaries and fast outbursts) that would require even more extended sampling to improve upon. However, the trade-off in time-sampling is areal sky coverage, which in turn would relatively reduced the areal coverage from 5.2 to 1.7 square degrees in going from 10-15 to 25-30 epochs as found in the FSVS sample.

Using two FSVS field groups, the distribution of variability over magnitude, color, and Galactic latitudes was investigated. The variability fraction as a function of $V$ magnitude (17.5-22.0 mag) is found to be 5-8\% in the FSVS in both the mid- and high galactic latitude field groups. The variability fraction is dominated by the $<0.05$ mag amplitude sources in field group 01 set (0.1 mag in field group 19) to $V \sim 19$ mag and is likely not due to the increasing photometric uncertainties as shown by the light curve simulations. These sources may represent a population of $\gamma$ Doradus stars. Beyond $V \sim 19$ mag, the variability fraction is dominated by sources with $<0.5 \mathrm{mag}$ amplitudes. The variability fraction as a function of $B-V$ shows a distinctive abundance of variable sources with $B-V<0.35$. The variability fraction redder than $B-V=0.35$ remains roughly constant at a few percent. The $B-V$ variability fraction plot divided up into rough $V$ magnitude 
bins reveals the $B-V$ peak occurs at all $V$ magnitudes at the longer time-scales, but the peak at hour time-scales peaks for sources fainter than $V \sim 19$ mag. Incorporation of the variable sources into a $B-V$, $V-I$ color-color plot indicates the majority lying along the Main Sequence colors. At the redder end of the Main Sequence the sources are likely active G-M dwarfs.

The results of the Bahcall Galaxy model (Bahcall \& Soneira 1986) predict 8-14\% of the stars as evolved off the Main Sequence, thus, it may be reasonable to associate the $B-V$ peak sources as RR Lyrae. A study of RR Lyrae stars in the SDSS by Ivezić et al. (2000) has found 148 candidates out of 100 sq. deg. to r' 21 mag. If the bluer sources are truly pulsating stars, with $V>20 \mathrm{mag}$, then many of the sources will be near the edge of the halo (Ivezić et al. 2000). However, Ivezić et al. (2000) note that $\delta$ Scuti stars (Population I type) will begin to disappear around $\mathrm{r}^{\prime}<13 \mathrm{mag}$ for galactic latitudes $>30^{\circ}$ and the remaining class of SX Phe (Population II) are only about $10 \%$ of $\delta$ Scuti population. Thus, to $V \sim 20$ mag, the pulsating variables are most likely RR Lyrae stars. Another possible source of variability from A to early $\mathrm{K}$ spectral type are the W UMa eclipsing binaries with an estimated space density of $2.5 \times 10^{-5} \mathrm{pc}^{-3}$ (i.e., $\sim 1$ out of every 500 A to early K star; Sterken 1996). There is also evidence for a population of newly classed $\gamma$ Doradus stars (F type dwarfs with g-mode pulsation periods ranging from 0.4-3 days and amplitudes to $V \sim 0.1$ mag.; Kaye et al. 1999) represented by the low amplitude fraction of sources in field group 01 that disappears by $V \sim 20$ mag. The bluer sources and outliers have also been found to be cataclysmic variables and even more so as quasars to fainter magnitudes from follow-up spectroscopy studies (Huber 2002; Howell et al. 2002; Clowes et al. 2006). There also exists a population of variable sources redder than $B-V=1.4$ (where the completeness in color also begins to degrade). However, a much larger population of variable sources beyond a $B-V$ of 1.4 (early M dwarf spectral type) is found in comparison to Everett et al. (2002) and is not surprising as the FSVS samples a much larger volume at its magnitude limits. Over the redder end of the color-color plot, G-M active dwarfs will likely contribute to the variability fraction due to starspots, chromospheric activity, and rotational effects. It is also possible for supernovae to be a constituent in the variability fractions. However, current rate estimates from other wide-field surveys predict on order of only a small few in an FSVS field group spread over the color range (Ho et al. 2001). With the relatively limited week time-scale sampling and single following year revisit without any immediate follow-up studies, it will be difficult to conclusively identify them.

Future work investigating the FSVS fields with the best time-sampling may be able to distinguish between short orbital modulation (W UMa, CVs) and pulsational sources (RR Lyrae, $\delta$ Scuti/SX Phe). However, the overall number of sources found to be variable range from $\sim 30-70$ per $V$ magnitude bin (15-35 in the bluer $B-V$ peak), providing an excess of sources even considering the populations of RR Lyrae stars, $\delta$ Scuti/SX Phe stars, W UMa binaries, and the smaller contributions from CVs and quasars. In future follow-up of these variable source, matching to SDSS colors may provide some separation of source types (Fan 1999), but better time-sampled photometry will provide the best identification and a data set to study the astrophysical parameters of the stellar sources (i.e., their periodicity).

We wish to thank the anonymous referee for the helpful comments on the manuscript. We are very grateful to Paul Groot, Paul Vreeswijk, and Gijs Nelemans in their tireless efforts leading to the public data release used here and in memory of Jan van Paradijs who fostered the original FSVS program design. MEH was partially supported by a NASA/Space Grant Fellowship, NASA Grant \#NGT-40008 and \#NGT400102, and some work was performed under the auspices of the U.S. Department of Energy, National Nuclear Security Administration by the University of California, Lawrence Livermore National Laboratory under contract No. W-7405-Eng-48. The FSVS is part of the INT Wide Field Survey. The INT is operated on 
the island of La Palma by the Isaac Newton Group in the Spanish Observatorio del Roque de los Muchachos of the Instítuto de Astrofísica de Canarias. 


\section{REFERENCES}

Bahcall, J. N., \& Soneira, R. M. 1984, ApJL, 238, 17

Bahcall, J. N., \& Soneira, R. M. 1980, ARA\&A, 84, 86

Becker, A. C., Rest, A., Stubbs, C., et al. 2004, in IAU Symp. 225, Impact of Gravitational Lensing on Cosmology, ed. Y. Melber \& G. Meylan (Cambridge: CUP), 34

Bessel, M. S. 1979, ApJS, 40, 1

Chen, B., et al. 2001, ApJ, 553, 184

Clowes, R. G., et al. 2006, in prep.

Cox, A. N. 2000, Allen's Astrophysical Quantities, 4th Edition (New York: AIP Press-Springer)

Everett, M. E., Howell, S. B., van Belle, G. T., \& Ciardi, D. R. 2002, PASP, 114, 656

Fan, X. 1999, AJ, 117, 2528

Green, R. F., Schmidt, G. D., \& Liebert, J. 1986, ApJS, 61, 305

Grenon, M. 1993, in ASP Conf. Ser. 40, Inside the Stars, ed. W. W. Weiss \& A. Gaglin (San Francisco: ASP), 693

Groot, P. J., Vreeswijk, P. M., Huber, M. E., Everett, M. E., Howell, S. B., Nelemans, G., van Paradijs, J., van den Heuvel, E. P. J., Augusteijn, T., Kuulkers, E., Rutten, R. G. M., \& Storm, J. 2003, MNRAS, 339,427

Ho, W. C. G., Van Dyk, S. D., Peng, C. Y., et al. 2001, PASP, 113, 1349

Howell, S. B., Mason, E., Huber, M., Clowes, R. 2002, A\&A, 395, 47

Huber, M. E. 2002, PhD Thesis, U. of Wyoming

Huber, M. E., Everett, M. E. et al. 2006a, in prep.

Huber, M. E., Howell, S. B., et al. 2006b, in prep.

Ivezić, $\check{Z}$, et al. 2000, AJ, 120, 963

Kaye, A., et al. 1999, PASP, 111, 840

Mathis, J. S. 1990, ARA\&A, 28, 37

Neese, C. 2002, private communication

Reid, I. N., \& Hawley, S. L. 2000, New Light on Dark Stars (London, Heidelberg: Springer-Verlag/Praxis)

Söchting I.K., Huber M., Howell, S.B., Clowes R.G. 2006, MNRAS, submitted

Stellingwerf, R. F. 1978, ApJ, 224, 953

Sterken, C. 1996, Light Curves of Variable Stars, ed. C. Sterken \& C. Jaschek (Cambridge: CUP) 
Tyson, A., \& Angel, R. 2000, in ASP Conf. Ser. 232, The New Era of Wide Field Astronomy, ed. R. G. Clowes, A. J. Adamson, \& G. E. Bromage (San Francisco: ASP), 284

Vivas, A. K., Zinn, R., Abad, C., et al. 2004, AJ, 127, 1158

Zaggia, S., et al. 1999, A\&ASS, 137, 75 

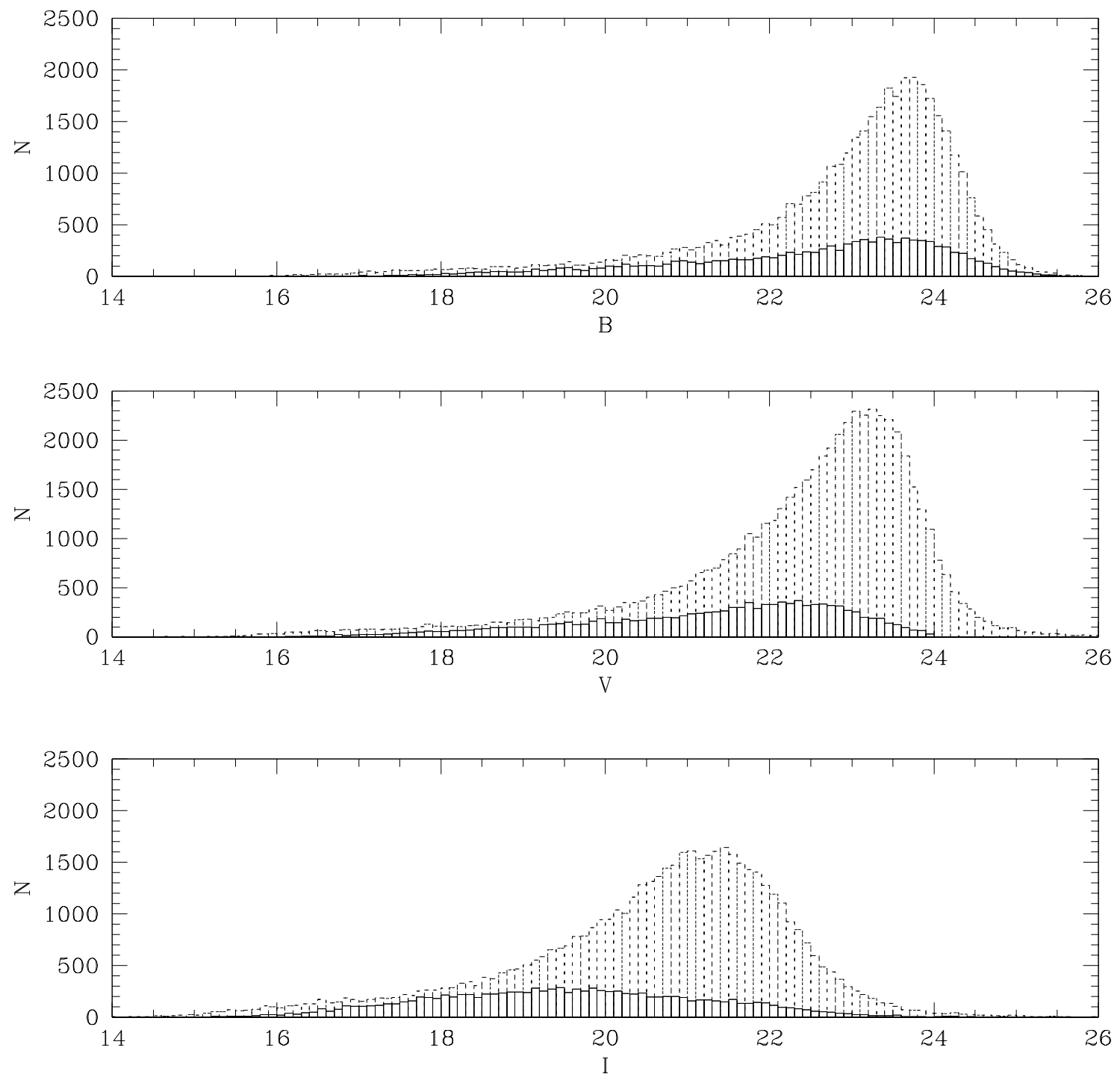

Fig. 1.- Dashed line histograms represent all sources individually detected in $B, V$, and $I$ for FSVS field group 01 . Solid line histograms represent point sources detected in $B, V$, and $I$. The qualification as a point source relies on the coadded $V$ stellarity $>0.8$ irregardless of a $V$ magnitude limit. 


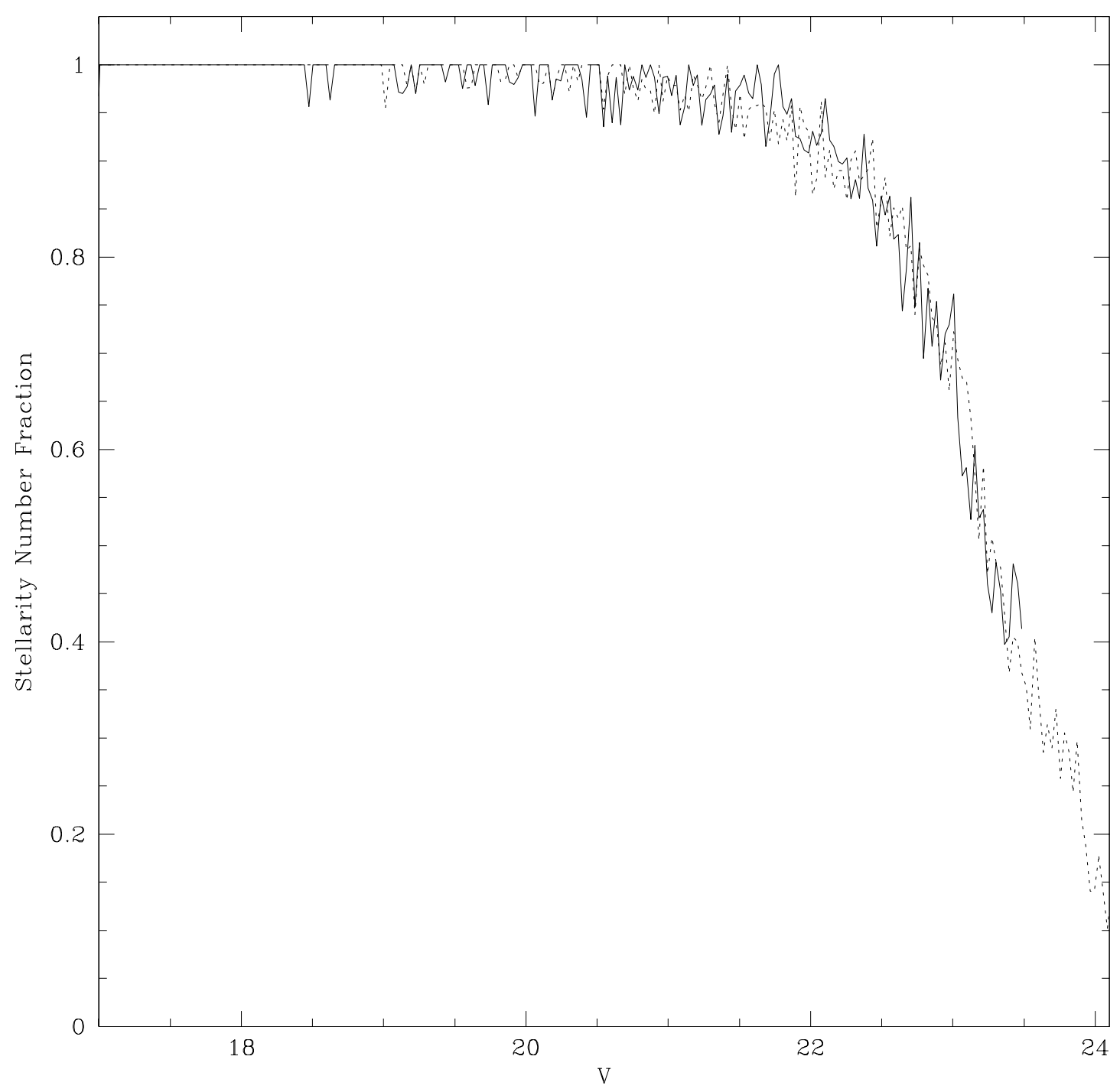

Fig. 2.- Plot of the number fraction for point sources as a function of magnitude. The solid line represents the number fraction of the number of sources with stellarity $>0.9$ divided by the number of sources with stellarity $>0.8$. The dashed line represents the number fraction of the number of sources with stellarity $>0.8$ divided by the number of sources with stellarity $>0.6$. At $V$ fainter than $\sim 23 \mathrm{mag}$, the stellarity value appears to lose reliability as the majority of the sources are below a stellarity of 0.9 . The same fraction of sources are found to also cross the point-extended threshold at 0.8 . At $V \sim 22 \mathrm{mag}, \sim 93 \%$ of the sources can still be considered strongly point-like. The turn up of the solid line is due to few sources having a stellarity $>0.8$ and being evenly distributed. 

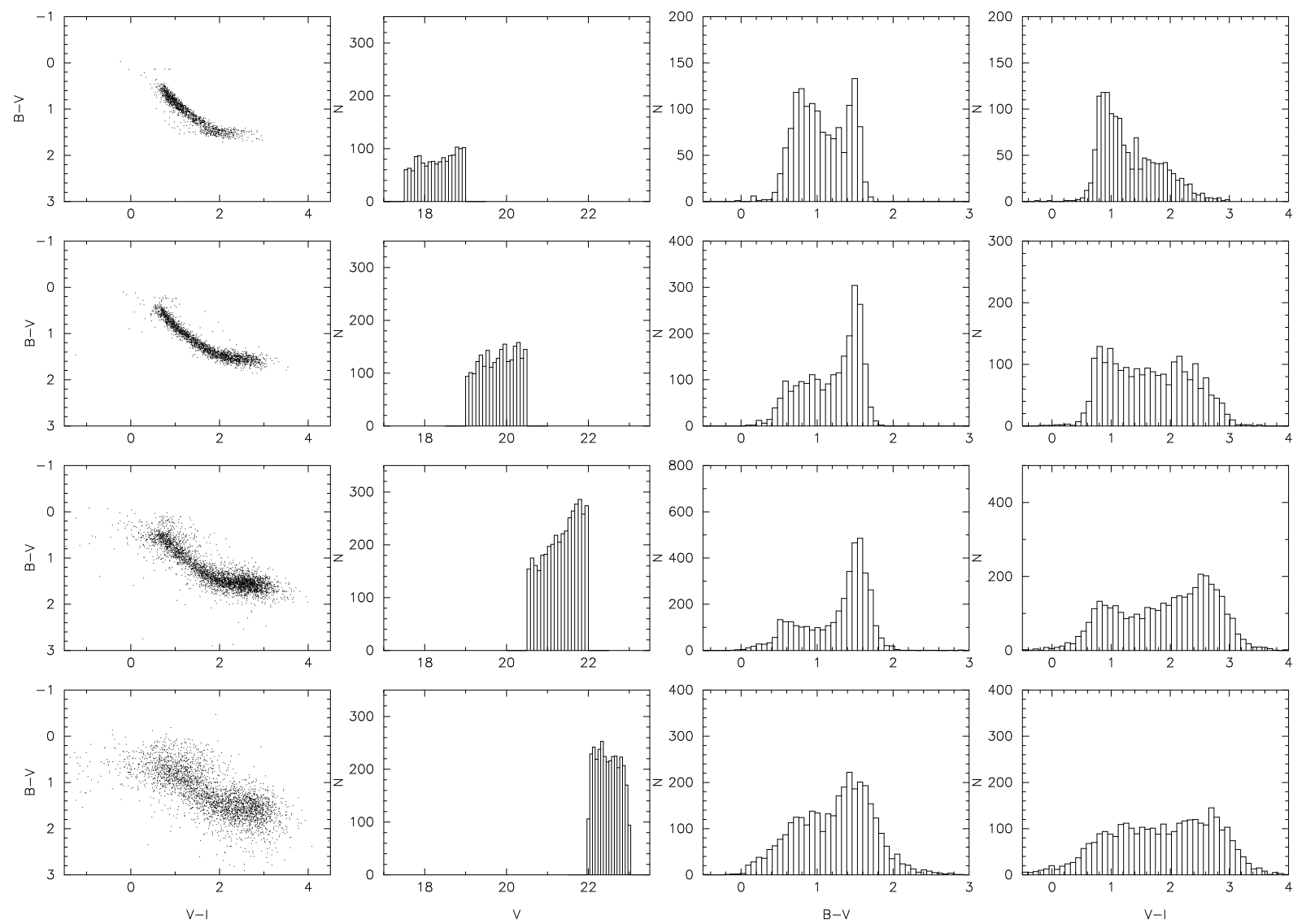

Fig. 3. - A presentation of the color space in the FSVS field group 01 divided into $V$ magnitude groups $V=17.5-19.0,19.0-20.5,20.5-22.0$, and 22.0-23.0 mag. 

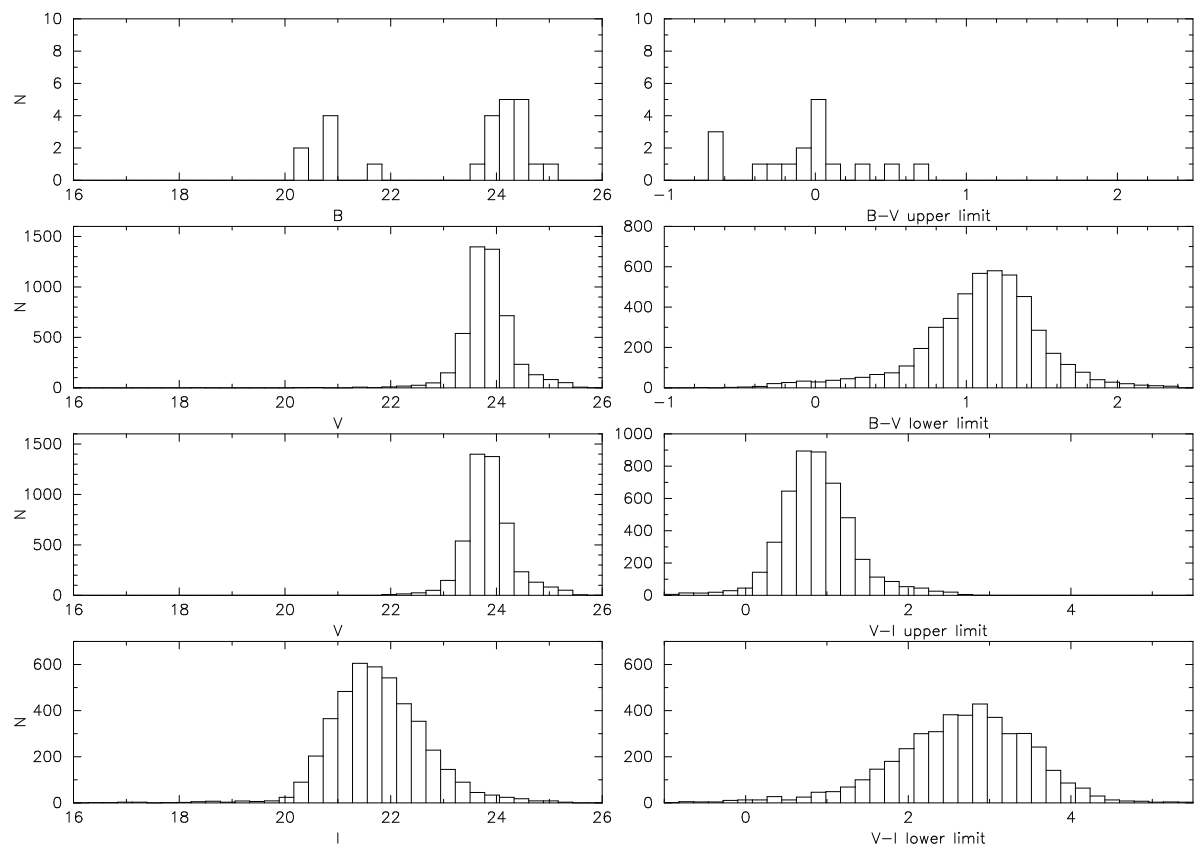

Fig. 4.- Histograms of sources detected in 1 filter for field group 01. The left side panels are the detections in the single filter with the color limit shown on the right side. The few sources at brighter magnitudes, particularly in $B$, are specifically identified as junk detections. Two $V$ only plots (middle panels) are shown to illustrate the $B$ and $I$ color limit relation to the detection.
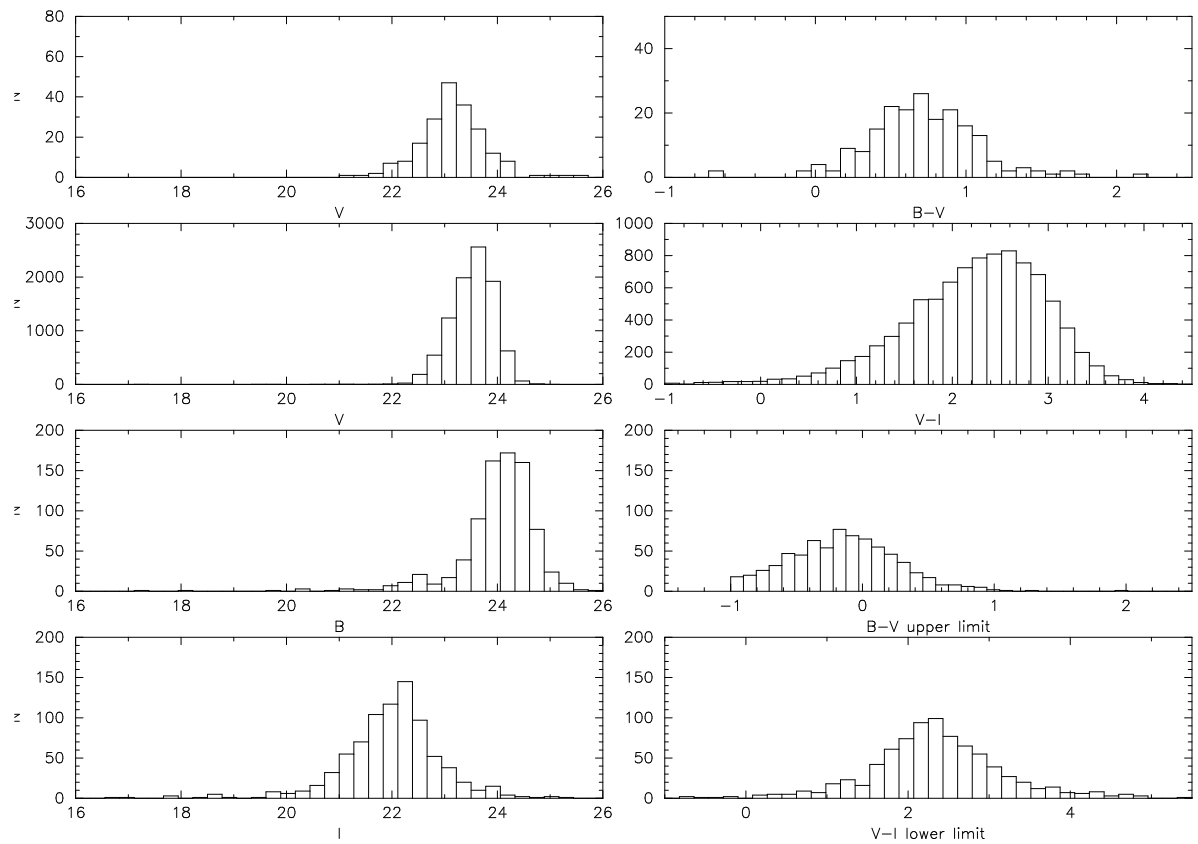

Fig. 5.- Histograms of sources detected in 2 filters for field group 01 . The top panel shows the $B V$ detected sources, the second from the top panel shows the $V I$ detected sources, and the lower two panels show the $B I$ detected sources with histograms of the $B$ and $I$ detected magnitudes. 

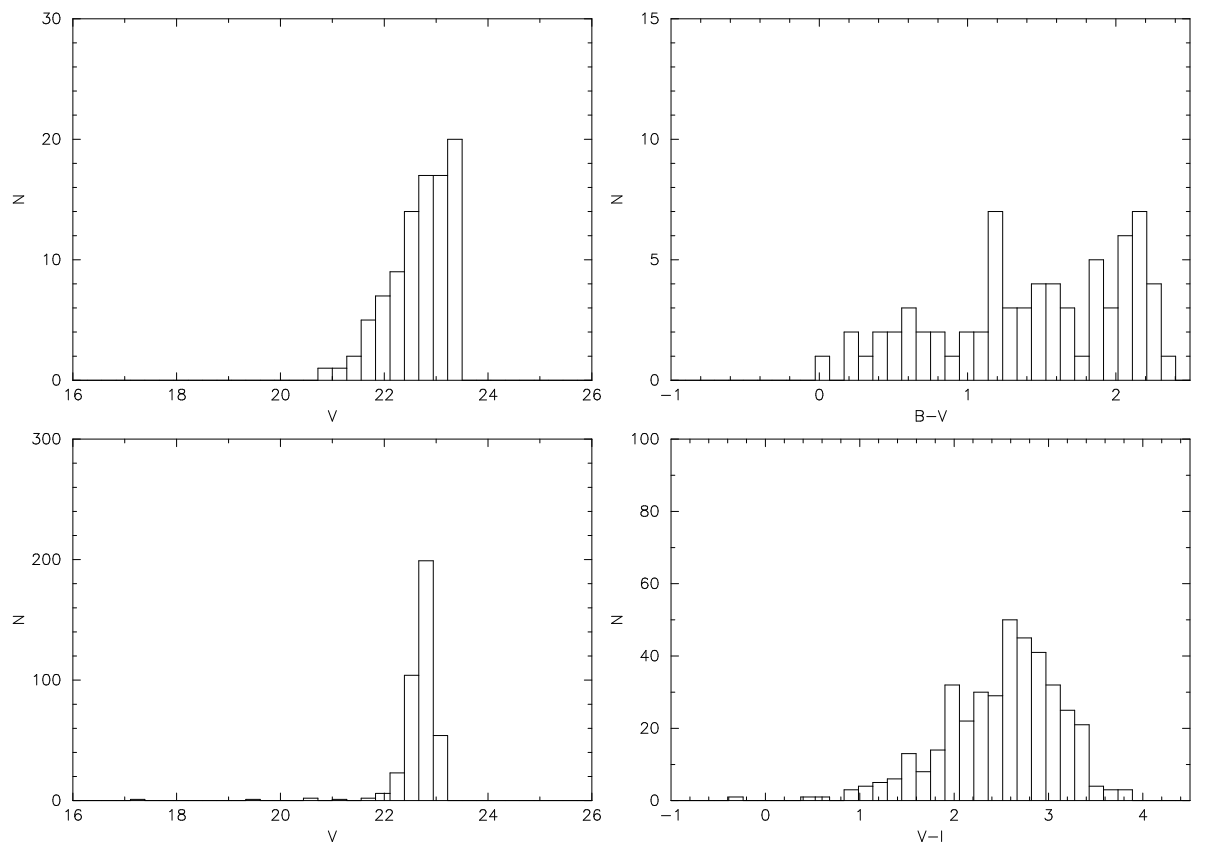

Fig. 6.- Histograms of extreme color point sources for field group 01. The top panel shows the $B V$ detections and the bottom panel shows the $V I$ detections, all with $V \leq 23$ mag. 

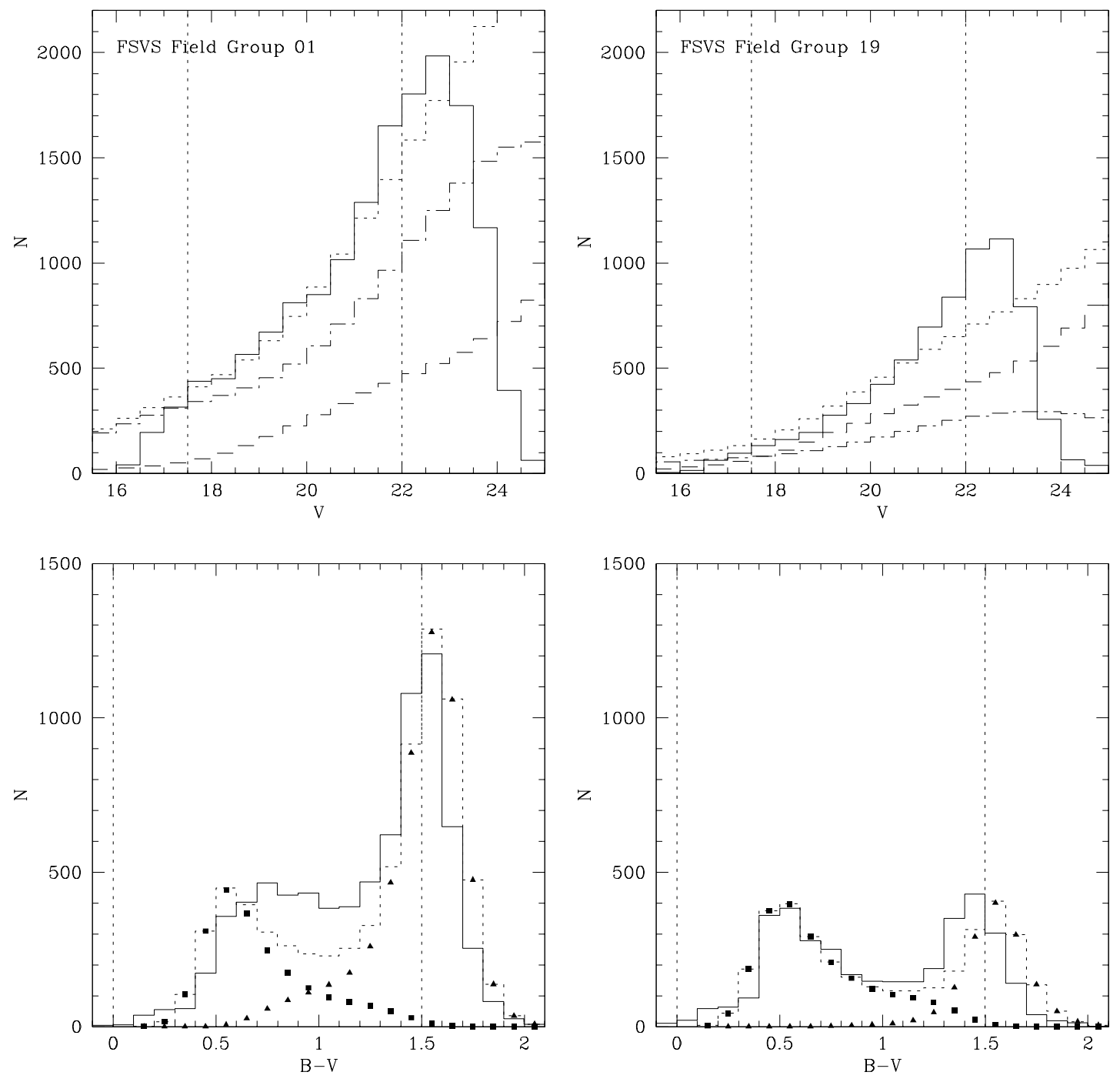

Fig. 7.- Histograms of the FSVS sources for FSVS field group 01 (left panels) and 19 (right panels). The top panels show the observed counts (solid line) as a function of $V$ magnitude in comparison to the expected total counts (dotted), expected disk (dot-dashed), and expected spheroid (dashed). The bottom panels show the observed counts over $B-V$ color space (solid line) in comparison to the expected total counts (dotted), expected disk (triangles), and spheroid (squares). The vertical dotted lines illustrate the completeness sample boundary, only sources with $V=17.5-22$ mag are shown in the $B-V$ plot. 

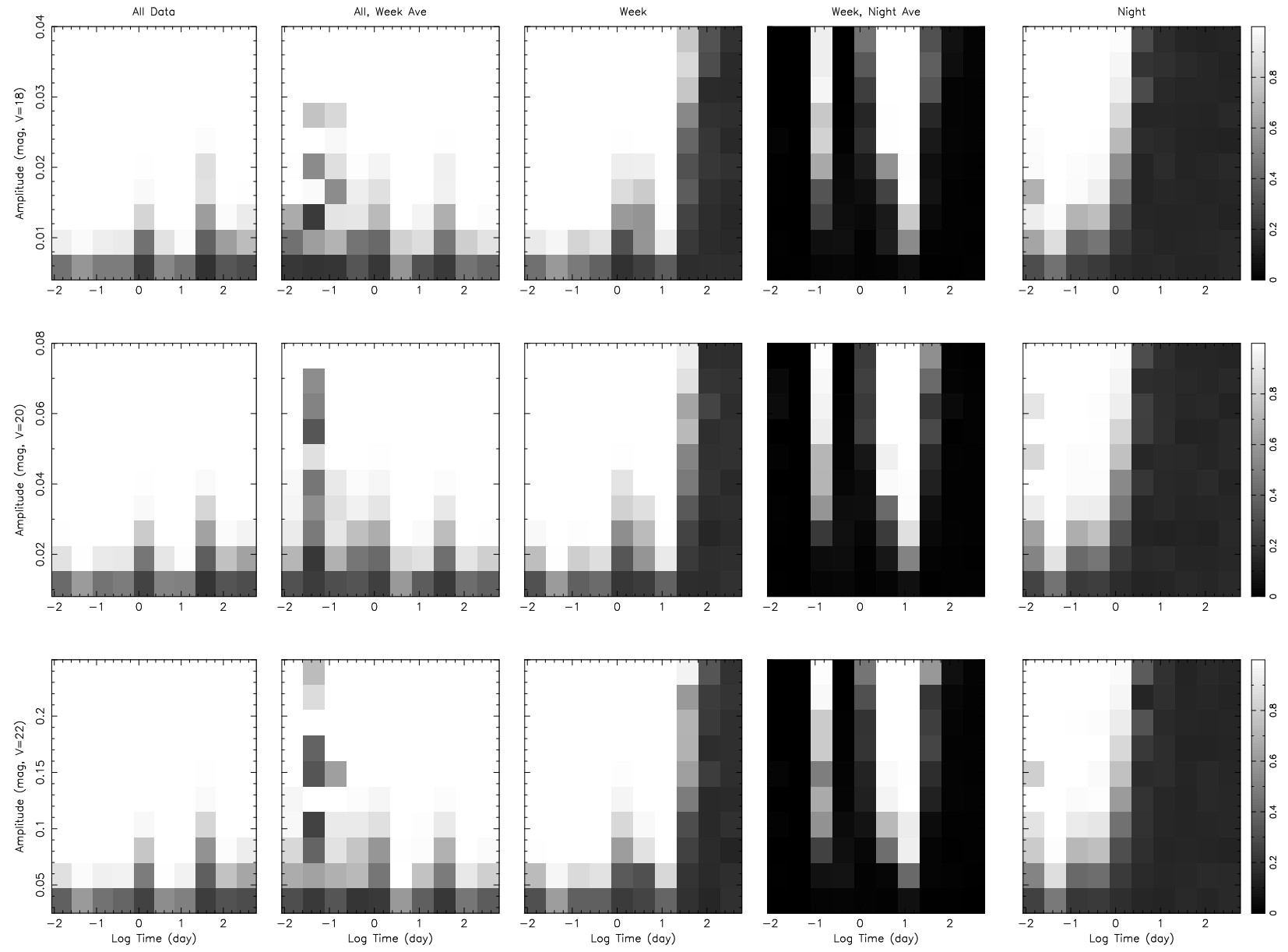

Fig. 8.- Image representations of the detection fraction of simulated variable sources using a threshold of $\mathrm{P}\left(\chi_{\nu}^{2}\right) \leq 10^{-2}$ for field group 01 sampling. Each panel gives the peak-to-peak amplitude of the sinusoid versus the Logarithm of the time in days ranging from 12 minutes to 600 days. Each horizontal set is taken at a similar mean magnitude that determines the photometric uncertainty and hence the amplitude range simulated. Each vertical set is a different sub-sample of the FSVS sampling set as described in Table 9. 

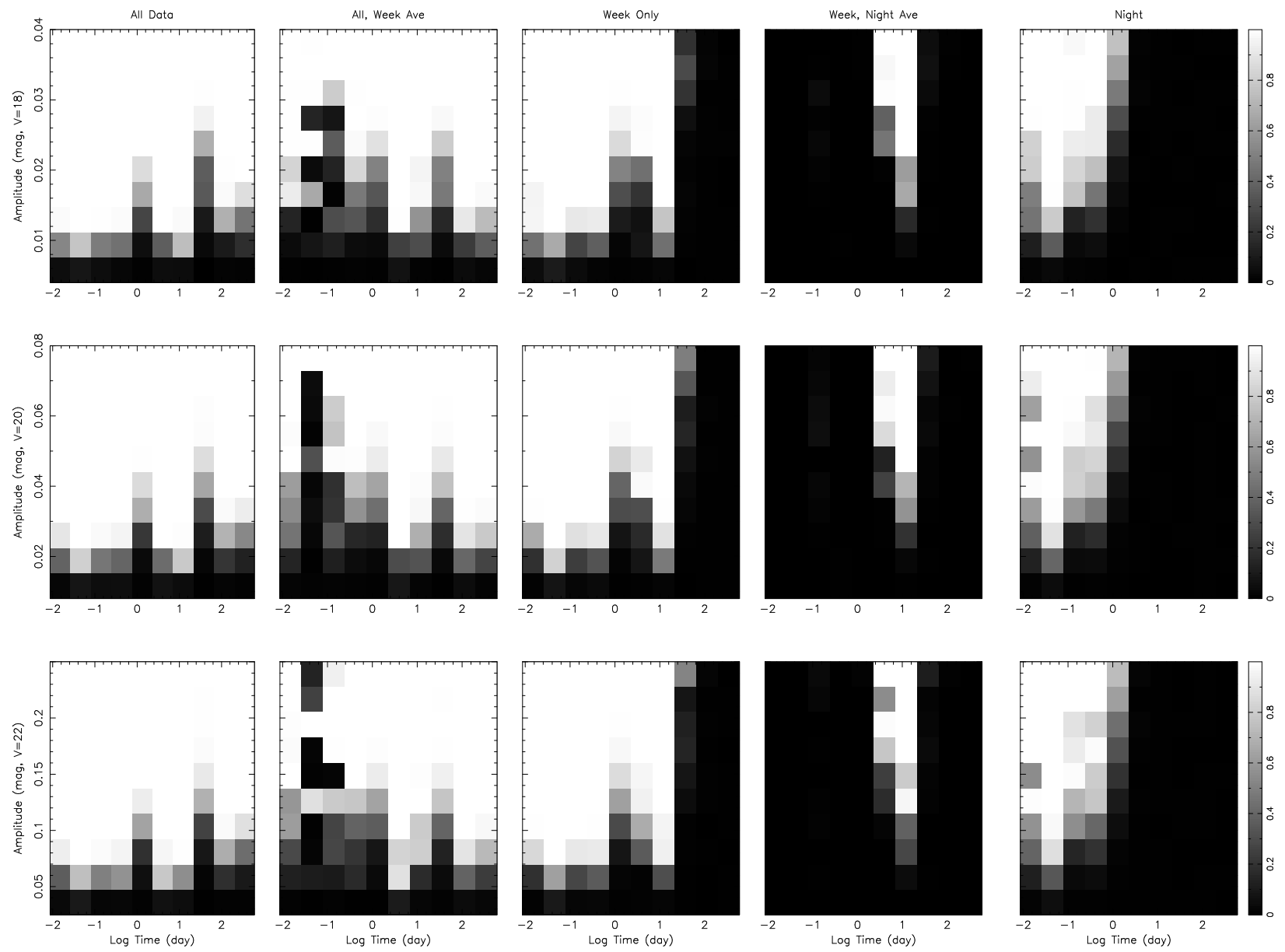

Fig. 9.- Same as described in Figure 8 except using a threshold of $\mathrm{P}\left(\chi_{\nu}^{2}\right) \leq 10^{-4}$. 

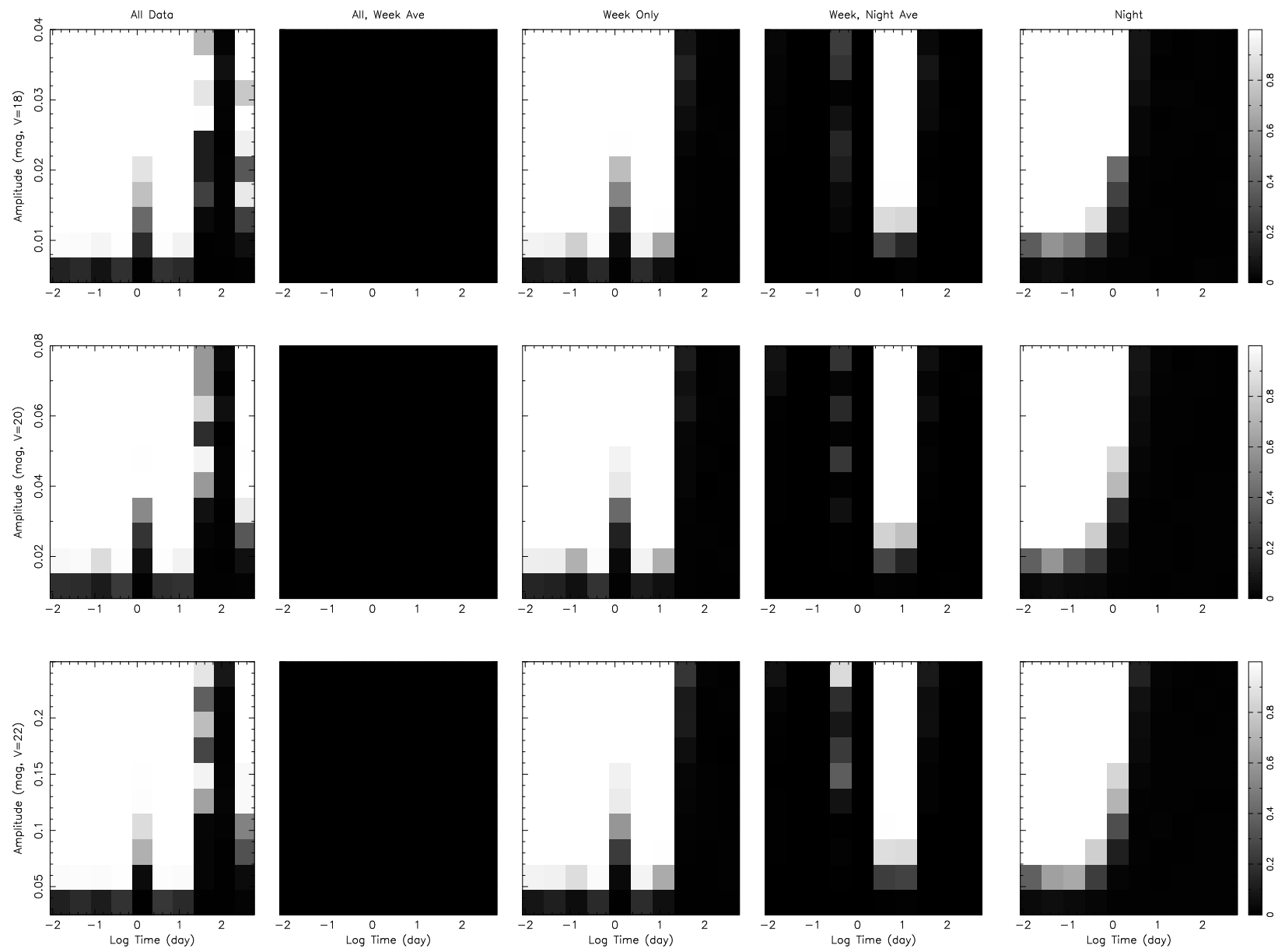

Fig. 10.- Same as described in Figure 8 except for field group 25 using a threshold of $\mathrm{P}\left(\chi_{\nu}^{2}\right) \leq 10^{-4}$. 

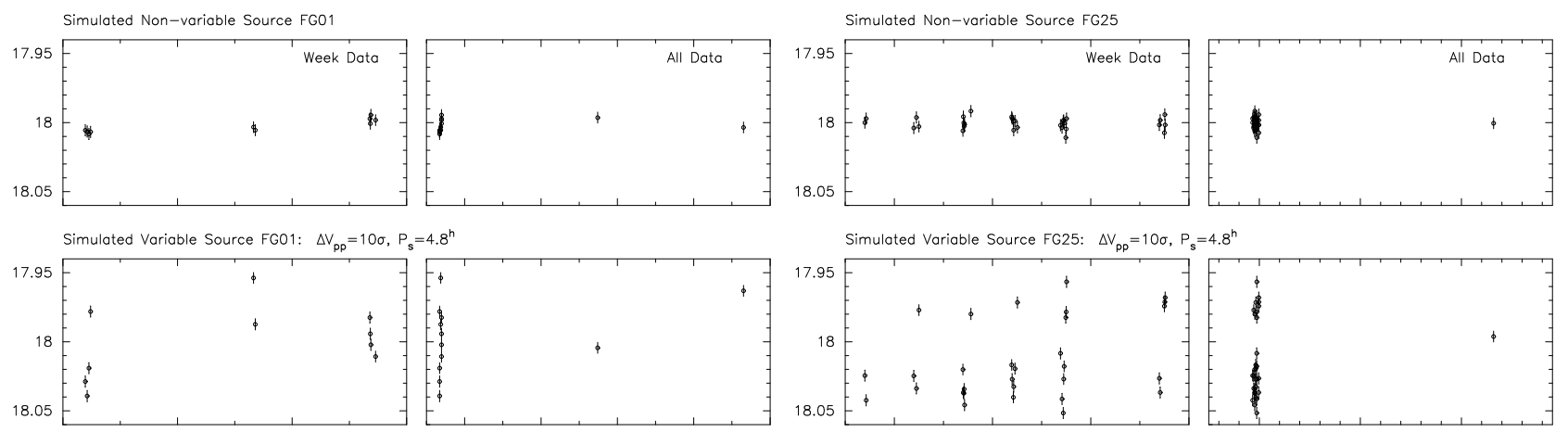

Simulated Variable Source FG25: $\Delta V_{P D}=10 \sigma, P_{s}=4.8$
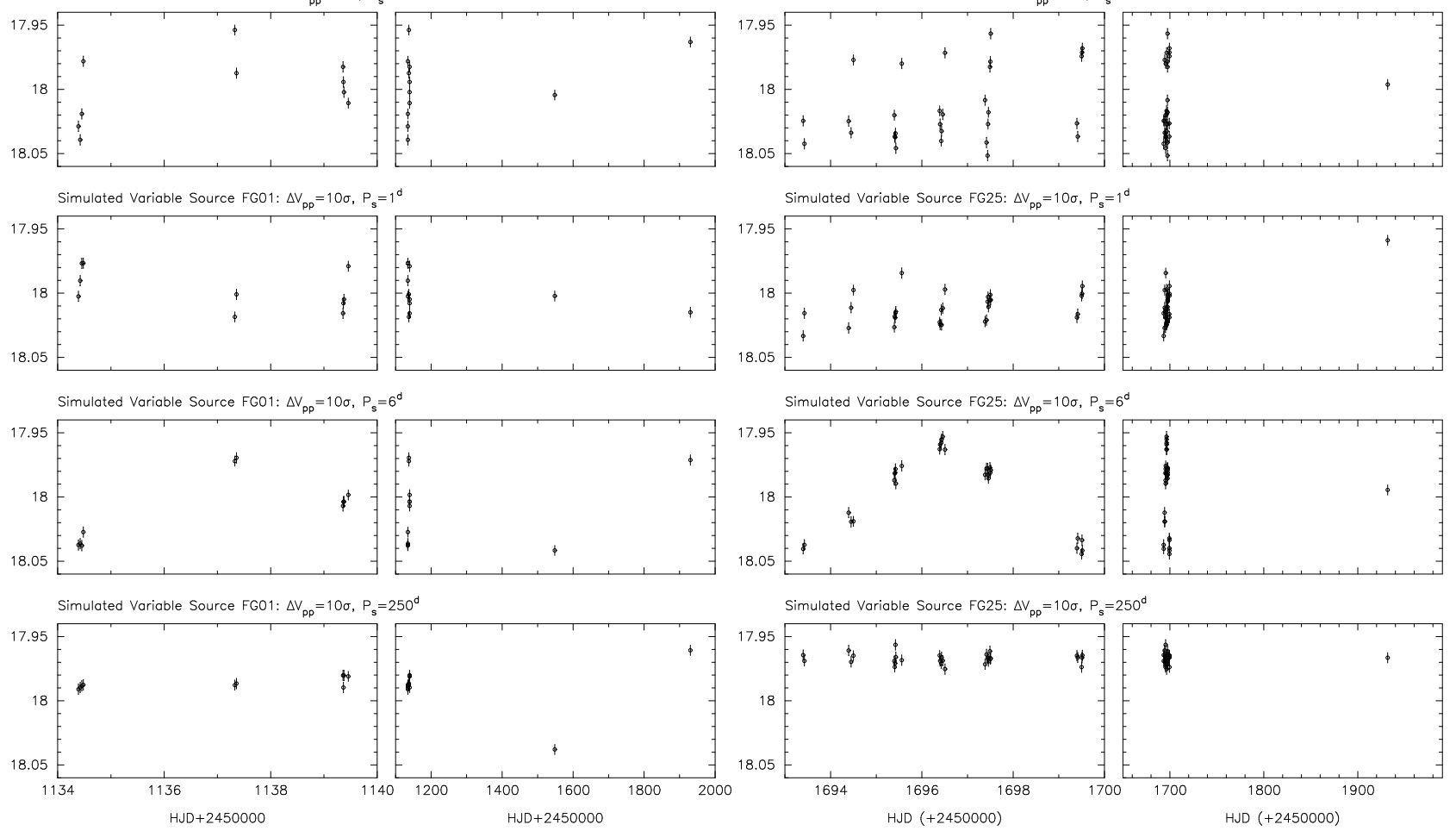

Fig. 11. - Simulated light curves sampled as FSVS field group 01 (left side panels) and 25 (right side panels) ranging from a non-variable simulation (top panel) to progressively longer sinusoidal periods (to the bottom panel). The observations spanning a week are shown in the left panel set and all the data is shown in the right panel set for field group 01 and 25. $\Delta V_{p p}$ is the peak-to-peak amplitude and $P_{s}$ is the period used to produce the light curve. 

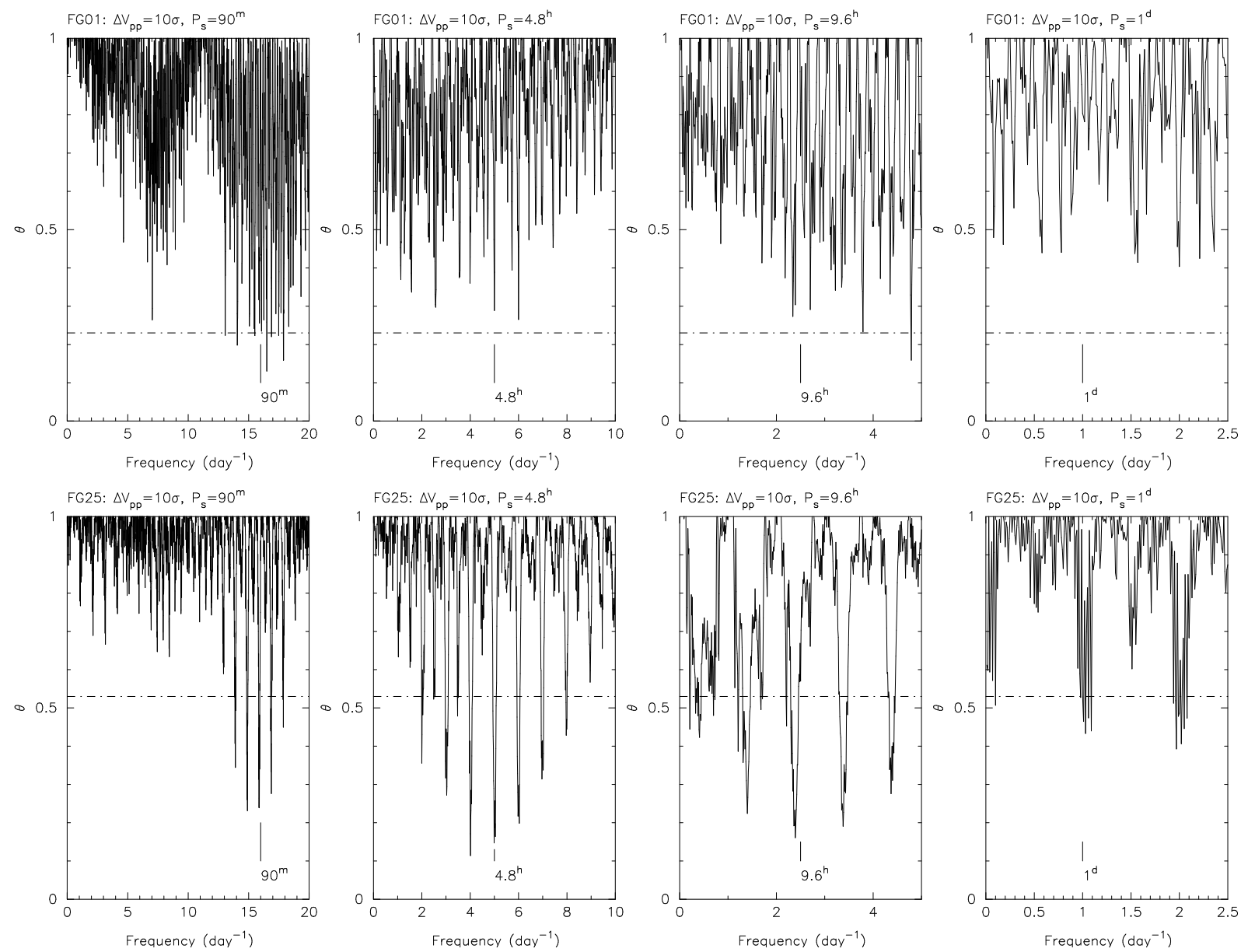

FG25: $\Delta V_{p p}=10 \sigma, P_{s}=1^{d}$

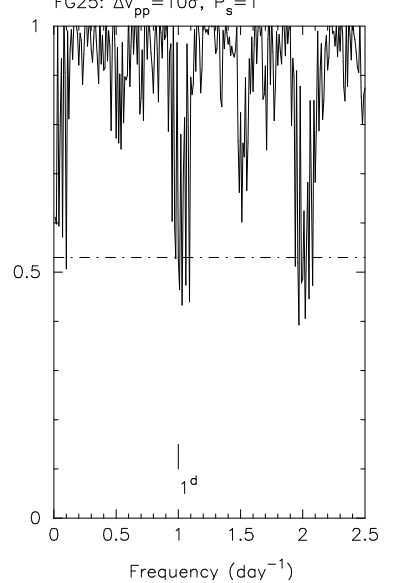

Fig. 12.- The eight thetagram plots represent the potential to determine periodicity in the FSVS survey data using simulated light curves. The dot-dash line represents the $95 \%$ confidence level for a given frequency, and the $\Delta V_{p p}$ and $P_{s}$ values are as in Figure 11. 

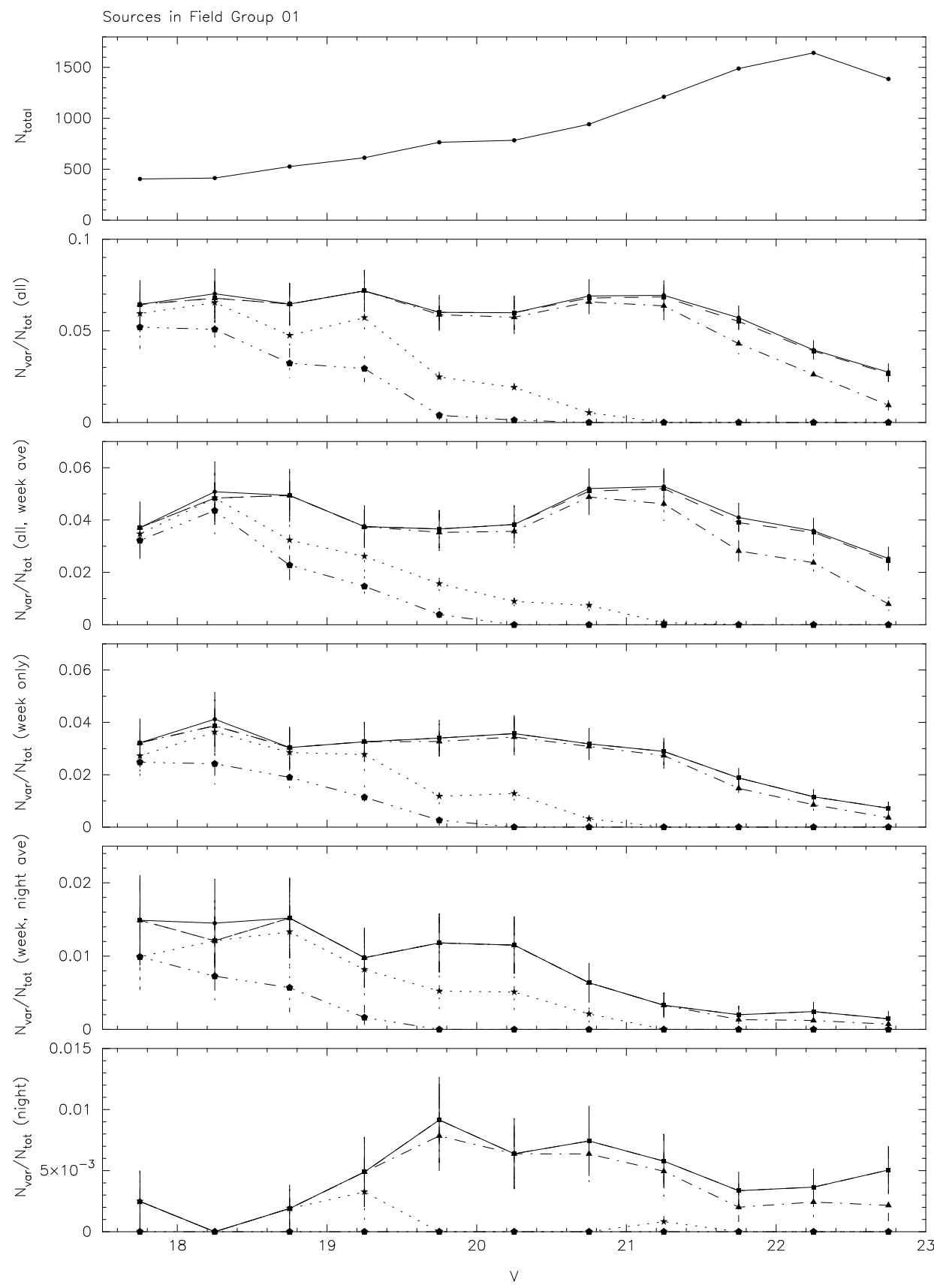

Fig. 13. - The variability fraction as a function of $V$ magnitude bins plotted for the different sub-samples in field group 01. Note that the completeness of the sample technically goes from $V=17.5-22.0$ mag. The top panel gives the total number of sources in each magnitude bin. The circles with solid lines are the fraction of all variables. The squares with dashed lines are the fraction of variables with amplitudes $<1.0$ mag. The triangles with dot-dashed lines are the fraction of variables with amplitudes $<0.5$ mag. The stars with dotted lines are the fraction of variables with amplitudes $<0.1 \mathrm{mag}$. The pentagons with three dot and dashed lines are the fraction of variables with amplitudes $<0.05$ mag. 

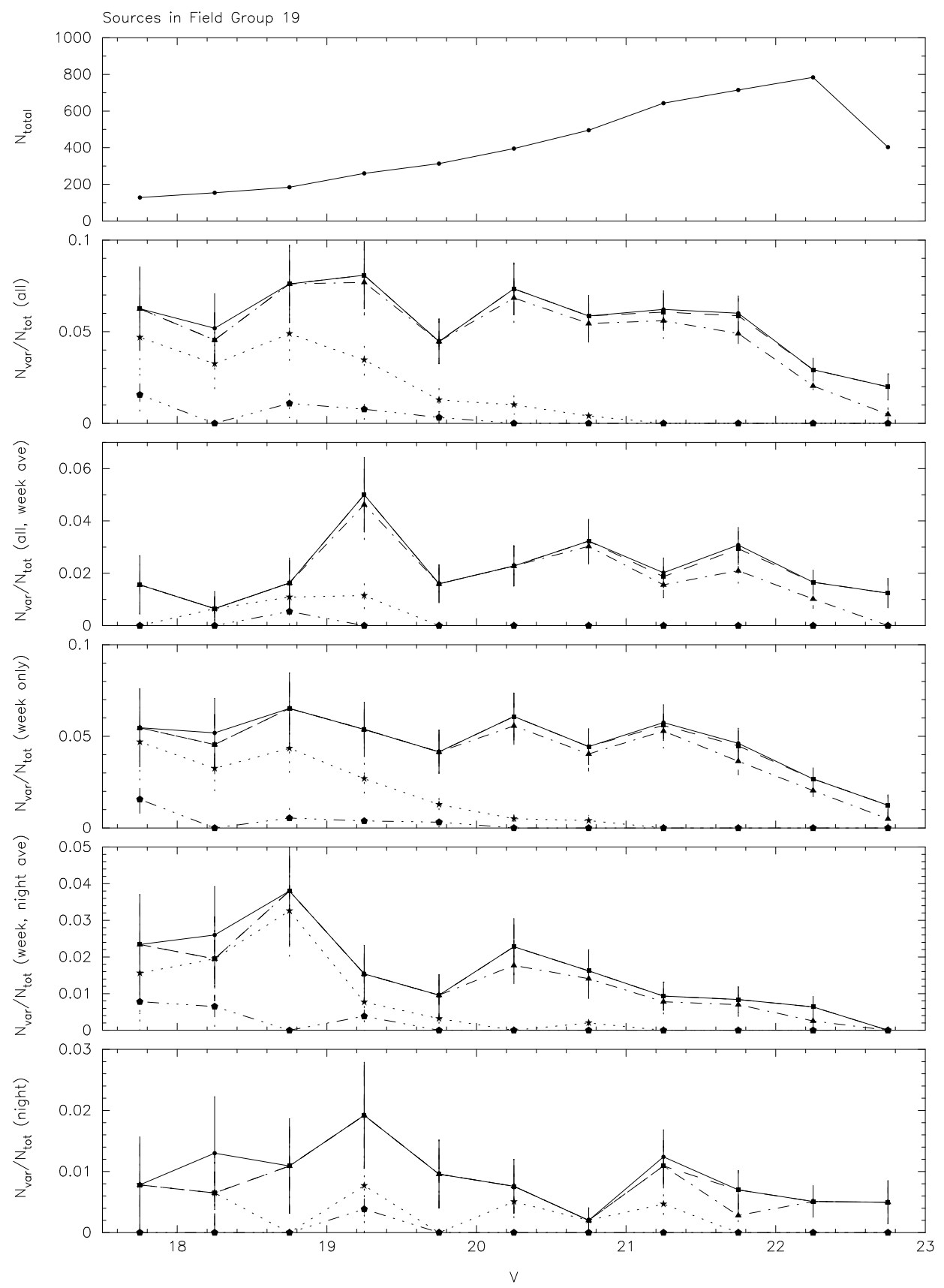

Fig. 14.- The variability fraction as a function of $V$ magnitude bins plotted for the different sub-samples in field group 19 with the same symbols as in Figure 13. 

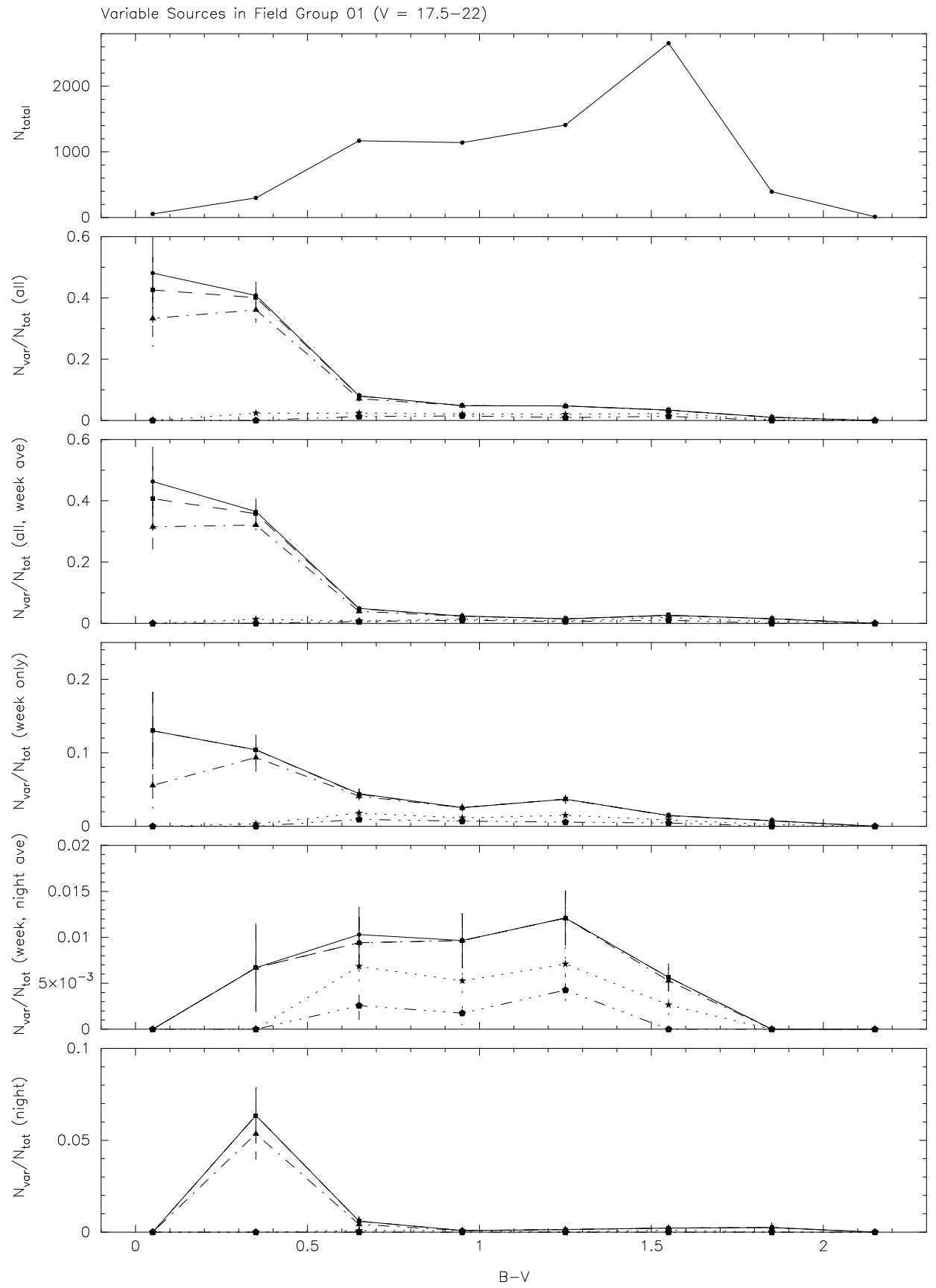

Fig. 15.- The variability fraction as a function of $B-V$ color bins for a $V=17.5-22$ mag plotted for the different sub-samples in field group 01. The symbols are the same as in Figure 13. 

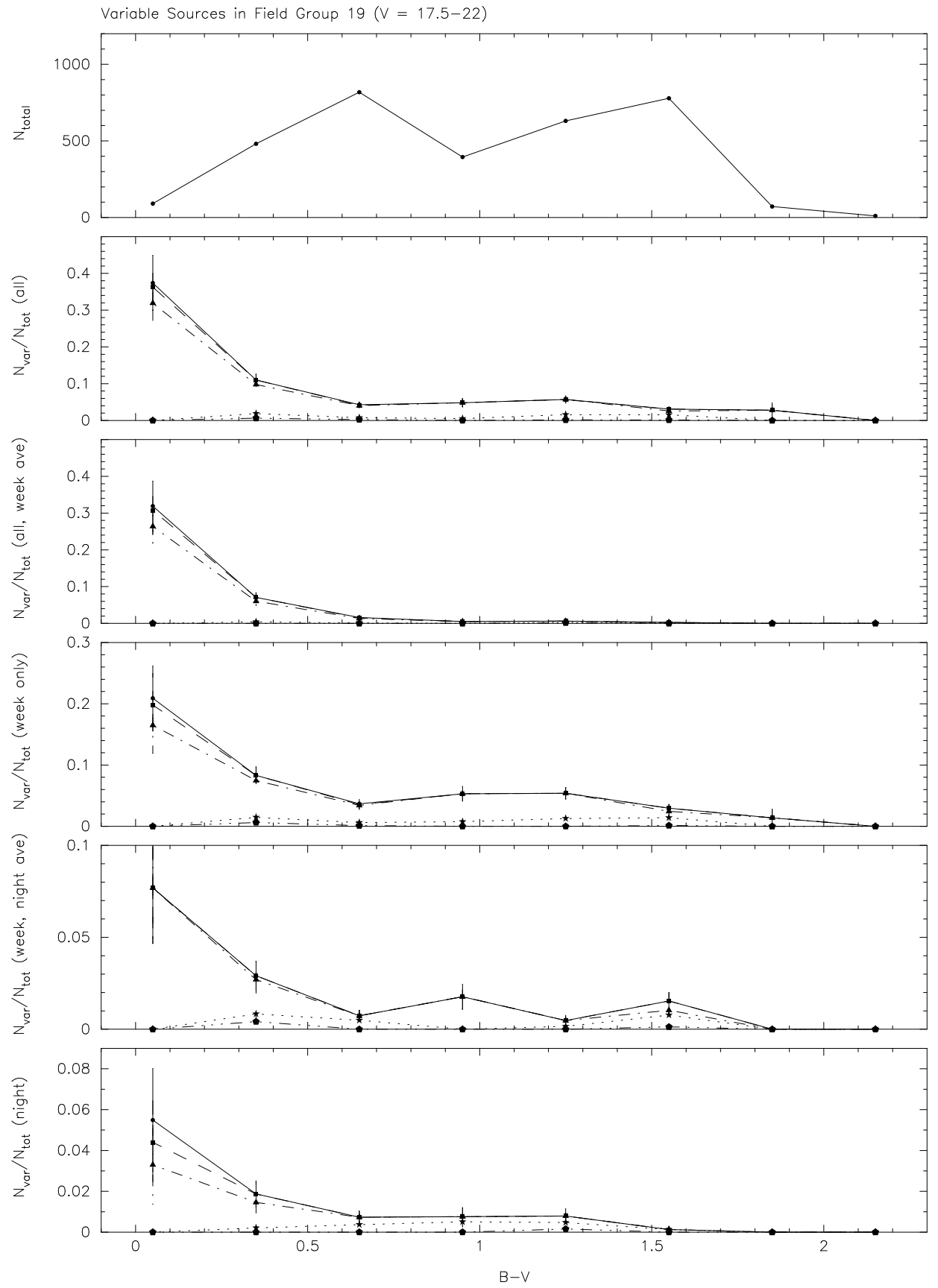

Fig. 16. - The variability fraction as a function of $B-V$ color bins for a $V=17.5-22$ mag plotted for the different sub-samples in field group 19. The symbols are the same as in Figure 13. 
Table 1. FSVS Fields in Contiguous Field Groups

\begin{tabular}{lccccc}
\hline \hline Field Group & Field \# & $l\left(^{\circ}\right)$ & $b\left(^{\circ}\right)$ & Sq. Deg. & \# Obs. \\
\hline 01 & $01-06$ & 105 & -33 & 1.73 & $12-15$ \\
19 & $19-24$ & $220-360$ & +90 & 1.73 & 13 \\
25 & $25-26$ & 282 & +89 & 0.58 & $29-30$ \\
\hline
\end{tabular}

Table 2. FSVS Field Group Color Sample

\begin{tabular}{lccccccccc}
\hline \hline $\begin{array}{c}\text { Field } \\
\text { Group }\end{array}$ & $\begin{array}{c}V \\
\text { Bright }\end{array}$ & $\begin{array}{c}V \\
\text { Faint }\end{array}$ & $\begin{array}{c}V \text { Star } \\
\text { Limit }\end{array}$ & $\begin{array}{c}V \text { Number } \\
\text { Peak }\end{array}$ & $\begin{array}{c}B \\
\text { Bright }\end{array}$ & $\begin{array}{c}B \\
\text { Faint }\end{array}$ & $\begin{array}{c}B \text { Number } \\
\text { Peak }\end{array}$ & $\begin{array}{c}B-V \\
\text { Range }^{\text {a }}\end{array}$ & $\begin{array}{c}N_{p t} \text { Sources } \\
\text { Adopted }\end{array}$ \\
\hline 01 & 17.4 & 24.2 & 23.0 & 22.4 & 17.5 & 24.9 & 23.6 & $0.1-1.1$ & 7510 \\
19 & 17.3 & 24.1 & 23.0 & 22.5 & 17.3 & 24.6 & 23.4 & $0.0-0.9$ & 3532 \\
25 & 17.5 & 24.0 & 23.0 & 22.6 & 17.6 & 24.7 & 23.4 & $0.1-0.9$ & 1264 \\
& & & & & & & & & \\
Mean & 17.4 & 24.1 & 23.0 & 22.5 & 17.5 & 24.7 & 23.5 & $0.1-1.0$ & $\ldots$ \\
Adopted $^{\mathrm{b}}$ & 17.5 & 22.0 & 23.0 & 22.0 & 17.5 & 23.5 & 23.5 & $0.0-1.5$ & $\ldots$ \\
\hline
\end{tabular}

${ }^{a}$ Determined as $(B-V)_{b r i g h t}$ and $(B-V)_{\text {peak }}$.

${ }^{\mathrm{b}}$ See text for adopted choices.

Table 3. FSVS Source Loss Percentages

\begin{tabular}{lccc}
\hline \hline \multicolumn{1}{c}{ Type of Source Loss } & $\%$ & $\%$ & $\%$ \\
& $(01)$ & $(19)$ & $(25)$ \\
\hline Stellarity Threshold $(V<22)$ & $<7$ & $<7$ & $<7$ \\
B, V, and $<80 \%$ Observations & 10 & 10 & 7 \\
Neighbors/Artifacts & $<1$ & $<1$ & $<1$ \\
IntraCCD Spacing & \\
& 3 & 3 & 3 \\
Internal Completeness & 99 & 99 & 99 \\
Adopted Completeness & $>83$ & $>83$ & $>85$ \\
& & & \\
\hline
\end{tabular}

${ }^{a}$ Not included in estimates (see text). 
Table 4. Extreme Color Source Detection Summary - Field Group 01 ${ }^{\mathrm{a}}$

\begin{tabular}{lccccccc}
\hline \hline Filter Detection & $B-V \leq$ & $B-V \geq$ & $V-I \leq$ & $V-I \geq$ & $\mathrm{N}$ & $\%_{\text {junk }}$ & $\%_{p t}^{\mathrm{b}}$ \\
\hline $\mathrm{B}$ & $0.52(10)$ & $\ldots$ & $\ldots$ & $\ldots$ & 16 & 0 & $\ldots$ \\
$\mathrm{V}$ & $\ldots$ & $0.52(10)$ & $1.47(13)$ & $\ldots$ & 4796 & 31 & $0.5^{\mathrm{c}}$ \\
$\mathrm{I}$ & $\ldots$ & $\ldots$ & $\ldots$ & $1.47(13)$ & 4320 & 8 & $\ldots$ \\
$\mathrm{B}, \mathrm{V}$ & $\ldots$ & $\ldots$ & $1.47(13)$ & $\ldots$ & 198 & 1.5 & 14.1 \\
$\mathrm{~V}, \mathrm{I}$ & $\ldots$ & $0.52(10)$ & $\ldots$ & $\ldots$ & 9244 & 2 & 4.6 \\
$\mathrm{~B}, \mathrm{I}$ & $0.52(10)$ & $\cdots$ & $\ldots$ & $1.47(13)$ & 856 & 25.8 & $\cdots$ \\
\hline
\end{tabular}

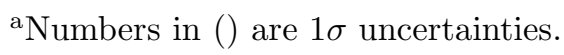

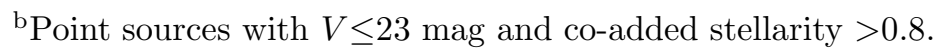

${ }^{\mathrm{c}}$ Sources noted as moving.

Table 5. Bahcall Model Parameter Summary

\begin{tabular}{lc}
\hline \hline Parameter & Value \\
\hline$M_{v}$ bright & -6.0 \\
$M_{v}$ faint & +16.5 \\
$m_{v}$ bright & 17.5 \\
$m_{v}$ faint & 22.0 \\
$\mathrm{~b}\left(^{\circ}\right)$ & $-33,+89-90$ \\
$\left.\mathrm{l}^{\circ}\right)$ & $105,220-360$ \\
Color Bins & $0.1 \mathrm{mag}$ \\
Halo Turnoff & $\mathrm{M} 13$ \\
\hline
\end{tabular}

Table 6. Bahcall Model Result Summary

\begin{tabular}{lcccccc}
\hline \hline $\begin{array}{c}\text { Field } \\
\text { Group }\end{array}$ & N Stars & \% Giants & $\begin{array}{c}\text { N Stars } \\
\text { (disk) }\end{array}$ & $\begin{array}{c}\text { \% Giants } \\
\text { (disk) }\end{array}$ & $\begin{array}{c}\text { N Stars } \\
\text { (spheroid) }\end{array}$ & $\begin{array}{c}\text { \% Giants } \\
\text { (spheroid) }\end{array}$ \\
\hline 01 & 7338 & 7.9 & 5212 & 0.0 & 2126 & 27.3 \\
19 & 3570 & 13.4 & 1419 & 0.0 & 2151 & 22.3 \\
25 & 1222 & 13.5 & 485 & 0.0 & 737 & 22.3 \\
\hline
\end{tabular}


Table 7. FSVS Field Group Time-Sampling Summary

\begin{tabular}{lccc}
\hline \hline $\begin{array}{c}\text { Field } \\
\text { Group }\end{array}$ & $\begin{array}{c}\text { Number } \\
\text { Observations }\end{array}$ & $\begin{array}{c}\text { Number in } \\
\text { Night }\end{array}$ & $\begin{array}{c}\text { Number } \\
\text { Year }\end{array}$ \\
\hline 01 & $12-15$ & $4-5,1-5,2-6$ & $2^{\text {a }}$ \\
19 & 13 & $3,3,5$ & 2 \\
25 & $29-30$ & $2,3,6,5-6,8,5$ & 1 \\
\hline
\end{tabular}

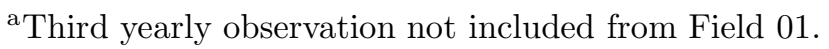

Table 8. FSVS Photometry Time Groups

\begin{tabular}{lll}
\hline \hline \multicolumn{1}{c}{ Sub-Group } & Observations Included & Points Averaged \\
\hline All & over 5 days +2 yrs & none \\
All, Week Ave & over 5 days +2 yrs & over 5 days \\
Week only & over 5 days & None \\
Week, Night Ave & over 5 days & within a night \\
Night & within a night & None \\
\hline
\end{tabular}

Table 9. Percentage of False Variability Detected in Non-variable Sources ( $V=18-22$ mag)

\begin{tabular}{lcccc}
\hline \hline \multicolumn{1}{c}{ Sub-Group } & $\begin{array}{c}\% \\
\mathrm{P}\left(\chi_{\nu}^{2}\right) \leq 10^{-1}\end{array}$ & $\begin{array}{c}\% \\
\leq 10^{-2}\end{array}$ & $\begin{array}{c}\% \\
\leq 10^{-3}\end{array}$ & $\begin{array}{c}\% \\
\leq 10^{-4}\end{array}$ \\
\hline All & $18.23(192)$ & $2.81(70)$ & $0.41(35)$ & $0.10(13)$ \\
All, Week Ave & $13.43(139)$ & $1.64(40)$ & $0.29(15)$ & $0.11(13)$ \\
Week & $17.45(140)$ & $2.76(79)$ & $0.31(23)$ & $0.06(5)$ \\
Week, Night Ave & $1.43(152)$ & $0.29(42)$ & $0.10(19)$ & $0.05(13)$ \\
Night & $18.67(744)$ & $2.38(103)$ & $0.32(19)$ & $0.07(7)$ \\
\hline
\end{tabular}

